\title{
Extracting partial decay rates of helium from complex rotation: autoionizing resonances of the one-dimensional configurations
}

\author{
Klaus Zimmermann ${ }^{1}$, Pierre Lugan ${ }^{1,2}$, Felix Jörder ${ }^{1}$, \\ Nicolai Heitz ${ }^{1}$, Maximilian Schmidt ${ }^{1}$, Celsus Bouri ${ }^{1,3}$, \\ Alberto Rodriguez ${ }^{1}$, and Andreas Buchleitner ${ }^{1}$ \\ 1 Physikalisches Institut, Albert-Ludwigs-Universität Freiburg, \\ Hermann-Herder-Str. 3, D-79104 Freiburg, Germany \\ 2 Institute of Theoretical Physics, Ecole Polytechnique Fédérale de Lausanne \\ EPFL, CH-1015 Lausanne, Switzerland \\ 3 Centre of Atomic Molecular Physics and Quantum Optics (CEPAMOQ), \\ Faculty of Science, University of Douala, Douala, P.O. Box 8580, Cameroon \\ E-mail: klaus.zimmermann@physik.uni-freiburg.de
}

\begin{abstract}
Partial autoionization rates of doubly excited one-dimensional helium in the collinear Zee and eZe configuration are obtained by means of the complex rotation method. The approach presented here relies on a projection of back-rotated resonance wave functions onto singly ionized $\mathrm{He}^{+}$channel wave functions and the computation of the corresponding particle fluxes. In spite of the long-range nature of the Coulomb potential between the electrons and the nucleus, an asymptotic region where the fluxes are stationary is clearly observed. Low-lying doubly excited states are found to decay predomintantly into the nearest singleionization continuum. This approach paves the way for a systematic analysis of the decay rates observed in higher-dimensional models, and of the role of electronic correlations and atomic structure in recent photoionization experiments.
\end{abstract}

\section{Introduction}

With only three constituents the helium atom is one of the simplest prototypes of a complex system in the realm of atomic physics, and it enjoys a long history of theoretical and experimental studies [1]. The complexity of helium is rooted in the long-range Coulomb interaction between the electrons and the nucleus, which gives rise to a wealth of interesting phenomena and challenging problems, related in particular to the mixed regular-chaotic phase space at the classical level [2], to high spectral densities below the double-ionization threshold [3], to the autoionization of doublyexcited states [4-7], or to the impact of electronic correlations in photoionization processes [8-12], to name a few essential aspects.

The main difficulty in the description of helium is the presence of the Coulomb interaction between the electrons, whereby the three-body dynamics cannot simply be modeled by two independent-electron problems [13]. The comparable magnitude of the Coulomb interaction between all three constituents also precludes simple perturbative schemes [14-16]. The complexity of the problem had been recognized early on, in the last century, when unsuccessful attempts were made to describe the spectrum of helium 
on the basis of early semiclassical quantization techniques. The electron-electron interaction lies at the heart of this failure, as it gives rise to mixed regular-chaotic structures in the high-dimensional phase space spanned by the motion of the electrons around the nucleus. Modern semiclassical theory has provided a better understanding about the link between quantum spectra and underlying classical dynamics $[1,14,17-$ 19]. In the case of helium, however, this link remains rather formal, as only part of the helium spectrum is so far understood in semiclassical terms. These limitations can be traced back to the fact that the high-dimensional classical phase space of the helium atom remains itself in its largest parts unexplored beyond the vicinity of one-dimensional configurations and the triple-collision point [9, 20], but also more fundamentally to the absence of an established semiclassical theory to describe the decay rates of an open quantum system such as helium [21].

At the level of the quantum spectrum of helium, the electron-electron interaction has two nontrivial consequences. First, while the Rydberg series of an independentelectron picture are scrambled, the excited states of helium are still organized in series [22]. The understanding of the symmetries underlying these spectral series and their classification according to new quantum numbers constitute a remarkable achievement of the group-theoretical [23, 24] and adiabatic approaches [13, 15, 25-29] developed by several authors over the last three decades. Second, all doubly excited states of helium are in fact resonance states, prone to various degrees of decay via autoionization [5, 7, 30]. Qualitatively, the formation of these decaying resonance states can be traced back to the Coulomb coupling of the doubly excited bound states of the independent-electron picture to neighbouring single-ionization continua. This picture, however, provides only little insight into how doubly excited states of helium autoionize, as the electron-electron coupling is nonperturbative. Unravelling the role of electronic correlations in few-electron atoms is in fact of prime interest to explain not only nonradiative decay via autoionization, but also photoionization processes or the nature of chemical bonds, as shown by recent and intense experimental and theoretical activity (see Refs. [8, 11, 31, 32] and references therein).

In the framework of the molecular adiabatic approach [15], the decay of doubly excited two-electron states can be understood as resulting from nonadiabatic transitions between adiabatic potential curves [1, 28, 33, 34]. To analyze the mechanism of such couplings, a set of propensity rules was established [29, 33, 35]. These propensity rules are approximate selection rules that account for the strong dominance of certain decay channels, based on the symmetries of the states described by the molecular adiabatic classification. While these rules were found to describe well the decay of resonances in low-lying series, their predictive power is reduced for higher excitations, as resonance series start to overlap and approximate quantum numbers are progressively lost for principal quantum numbers $N \geq 6$ [22, 34]. For such regimes where several decay channels may play comparable roles, resorting to tools allowing the quantitative analysis of partial decay rates of resonance states (i.e. branching ratios between decay channels) from first principles appears necessary. This need arises not only for autoionization processes, but also for the analysis of electron-impact ionization and photoionization.

Various numerical $a b$ initio approaches have been developed and intensively used over the past thirty years to study the consequences of electron-electron correlations on spectra and ionization processes in helium (for a review see Refs. [1,36] and references therein). Among those, the method of complex rotation [37-49] has established itself as a powerful technique allowing the computation of resonance positions and 
widths as well as ionization cross sections with unprecedented accuracy, including high-lying resonance series [7, 22, 50-53]. However, in contrast to time-dependent calculations [54-57] and other time-independent numerical approaches relying e.g. on $R$-matrix techniques [58-61], the Feshbach projection formalism [62], or close coupling methods [27, 63-66], which have been used successfully to compute partial ionization rates in regimes of moderate excitation [67,68] and close to the three-body breakup threshold [69], the application of the complex-rotation technique to helium has hitherto mostly been restricted to the calculation of total decay rates and cross sections [1]. A notable exception to this state of affairs for the three-body Coulomb problem is provided by a calculation of angle-resolved differential cross sections for the electronimpact ionization of hydrogen [70].

Early schemes to calculate partial decay rates within the framework of complex rotation have been devised in Refs. [71-74]. The key element in these proposals is that, while the complex eigenenergies obtained from complex rotation yields only the resonance positions and widths, the associated resonance wave functions (eigenvectors) may be used to compute transitions matrix elements [71], or projected onto channel wave functions [72-74] to deduce the associated partial decay rates. The above schemes rely on the use of the (square-integrable) complex-rotated resonance wave function, and require either an integration over the radial degree of freedom along which decay occurs [71, 72] or plane-wave asymptotics of the resonance wave function [73, 74], which precludes a straightforward application to long-ranged Coulomb potentials.

We employ here an alternative approach to calculate partial decay rates, which was initiated in Ref. [75] (a related approach was examined in Ref. [76]) and which relies on an inspection of the particles fluxes associated with the back-rotated resonance wave functions. With this method, we analyze the partial decay rates of atomic resonance states in two one-dimensional models of helium, namely the so-called Zee and $e Z e$ configurations, where the two electrons lie on the same or on opposite sides of the nucleus, respectively. Such collinear models of helium lack the angular degrees of freedom, but contain essential ingredients of the full-dimensional helium atom, such as autoionizing resonance states, overlapping series in the highly doubly excited regime, high spectral densities and an accumulation of series close to the doubleionization threshold. Moreover, these configurations correspond to invariant subspaces of classical motion in 3D helium [1]. The Zee subspace and its vicinity have been analyzed thoroughly (see e.g. Refs. [77, 78]) as it has been recognized, in particular, that they host pronounced resonances associated with stable islands in classical phase space, known as frozen-planet configurations [79]. Our approach for the calculation of partial decay rates may thus be useful not only for the quantitative analysis of fragmentation processes in strongly correlated few-electron systems, but also from the point of view of the semiclassical physics of mixed phase spaces [21].

This article is organized as follows. In section 2 we briefly review the spectral structure of collinear helium, and we present the analytical and numerical tools used for the description of resonance states within the framework of complex rotation. In section 3 we present our general scheme for the calculation of partial decay rates. In section 4 this procedure is used to analyze the decay of autoionizing doubly excited states of Zee and $e Z e$ helium. In section 5, ultimately, we summarize our results. 


\section{Wave functions of atomic resonance states in one-dimensional helium}

In the one-dimensional (1D) model of helium, the nucleus and the two electrons are constrained to move on one fixed line, with the electrons either both residing on one side of the nucleus $(Z e e)$, or on opposite sides $(e Z e)$. In the limit of an infinitely massive nucleus, and neglecting relativistic effects, the dynamics of the electrons with respect to the nucleus is governed by the Hamiltonian

$$
H=-\frac{1}{2} \frac{\partial^{2}}{\partial z_{1}^{2}}-\frac{1}{2} \frac{\partial^{2}}{\partial z_{2}^{2}}-\frac{Z}{z_{1}}-\frac{Z}{z_{2}}+\frac{\gamma}{\left|z_{2} \mp z_{1}\right|},
$$

where $z_{i}>0$ is the distance of electron $i$ from the nucleus, $Z=2$ is the Coulomb charge of the nucleus, $\gamma$ is a numerical parameter accounting for the strength of Coulomb interaction between the electrons ( $\gamma=1$ amounts to the natural interaction), and Hartree atomic units (a.u.) have been used: $e \equiv m_{e} \equiv \hbar \equiv 1 /\left(4 \pi \epsilon_{0}\right) \equiv 1$. With the latter, the length unit is the Bohr radius $a_{0}$ and the energy unit is the Hartree $(\sim 27.2 \mathrm{eV})$ [80]. The Hamiltonian $H$ acts on two-body wave functions $\psi\left(z_{1}, z_{2}\right)$ that are either symmetric (even) or antisymmetric (odd) under the exchange of coordinates. In the Zee case this symmetry originates from particle exchange, and corresponds to singlet and triplet spin states, respectively. In the $e Z e$ case, the symmetry arises from the invariance of $H$ under the combined action of particle exchange and central symmetry, i.e. reflection of all coordinates about the origin. Furthermore, irrespective of symmetry, the Coulomb potential between electrons demands a nodal line on the $z_{1}=z_{2}$ line of Zee configurations, effectively rendering the even and odd wave functions degenerate and identical, save for a sign in half of configuration space. As a consequence the configuration space may be reduced to the sector $z_{1}>z_{2}$, and we will hereafter simply talk about Zee helium without distinction of symmetry. In contrast, symmetric $e Z e$ states show an antinode on the $z_{1}=z_{2}$ line, so that the $e Z e$ states need to be distinguished according to symmetry. This symmetry has important repercussions on the physical properties of eigenstates as, in particular, the nodal $z_{1}=z_{2}$ line of odd $e Z e$ states leads to a vanishing density close to the triple collision point and consequently to an increased stability compared to the even states [81].

\subsection{Autoionization resonance states in helium}

In the absence of interaction between the electrons $(\gamma=0)$, the electronic dynamics is separable, and $H$ reduces to the sum of two hydrogen-like Hamiltonians, each of which gives rise to a Rydberg series of bound states and a continuum of Coulomb scattering states. The resulting two-electron spectrum is depicted in Fig. 1. Each bound state of the lowest-lying electron, labelled by the quantum number $N$, comes along with a Rydberg series $E_{N n}=E_{N}^{\mathrm{SIT}}-Z^{2} /\left(2 n^{2}\right)$ a.u., where $n \geq N$ (even, singlet) or $n>N$ (odd, triplet), and a continuum of singly-ionized helium states above the $N$-th singleionization threshold $E_{N}^{\mathrm{SIT}}=-Z^{2} /\left(2 N^{2}\right)$ a.u. For increasing $N$, these series converge to the double-ionization threshold $E^{\text {DIT }}=0$ a.u.

The inclusion of the Coulomb interaction between the electrons does not change the positions of the ionization thresholds, but strongly affects the discrete part of the spectrum. The discrete levels of the Rydberg series couple among themselves and to the ionization continua. As a result, all levels are shifted and the continuum coupling turns doubly excited bound states with $n \geq N>1$ into resonance states with a finite lifetime. Doubly excited states of $1 \mathrm{D}$ helium thus decay naturally via autoionization into singly ionized $\mathrm{He}^{+}$ions, without any perturbation by an external 


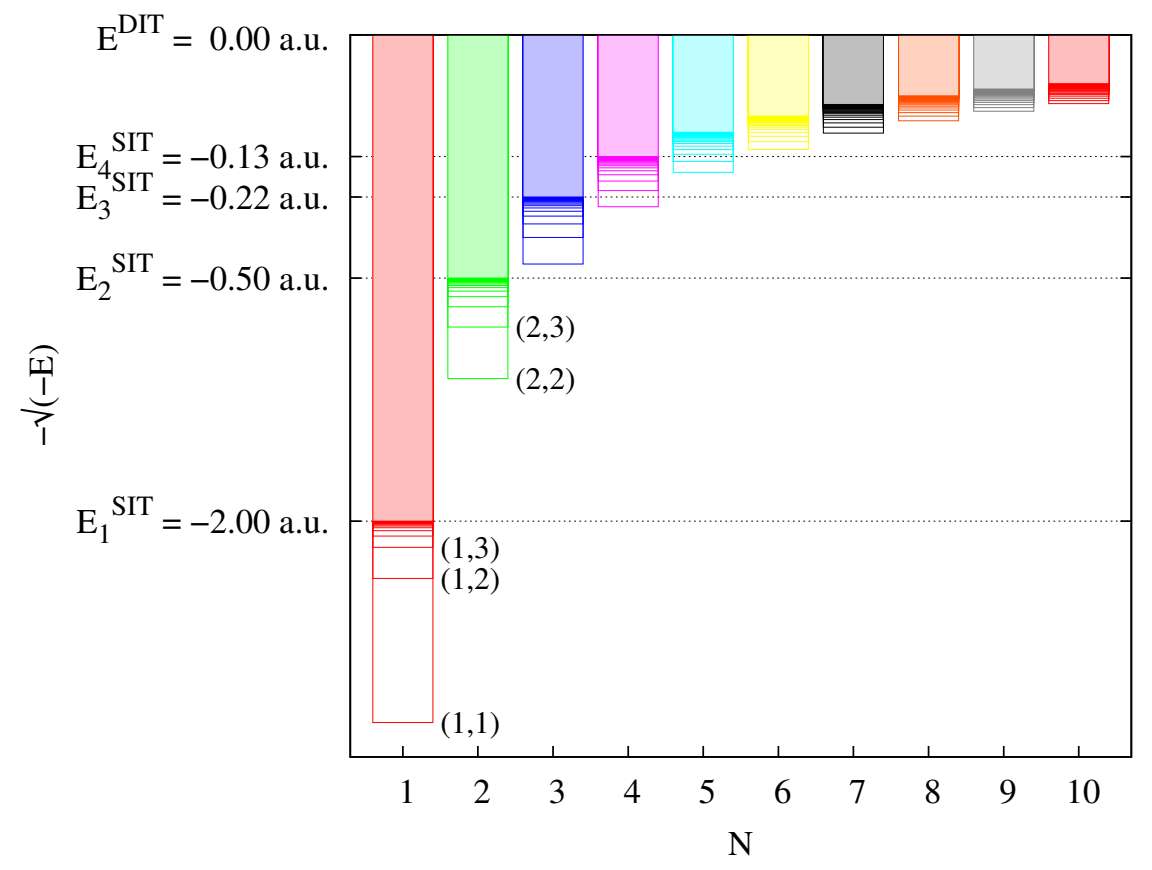

Figure 1. (Colour online) Schematic spectrum of 1D helium without electronelectron interaction. The quantum numbers of the two electrons are $N$ and $n$. For every fixed $N$ there is a Rydberg series with $n>N$ ( $n \geq N$ for even states) that converges to the respective single-ionisation threshold. Note that in the case of even (singlet) Zee configurations, the $n=N$ states disappear as soon as even a weak Coulomb potential is turned on between the electrons. To exemplify the notation, we explicitly label five states by $(N, n)$. The single-ionization thresholds of the first four series are marked. Note that the ground state of the second series is energetically already embedded in the continuum of the first. Note further, that in this uncoupled picture the series start to overlap already from $N=5$ onwards, i.e. the ground state of the fifth series is lower in energy, than the single-ionization threshold of the fourth. The single-ionization thresholds $E_{N}^{\mathrm{SIT}}=-2 / N^{2}$ a.u. and the double-ionization threshold $E^{\text {DIT }}=0$ a.u. are left unaffected by a nonvanishing electron-electron coupling.

field. This phenomenology also holds in higher dimensions [1, 4]. In particular, the Zee autoionization resonances discussed here arise as the 1D counterparts of the frozenplanet states known from higher-dimensional models of helium [79, 82], although the lifetimes of the latter are considerably shorter, due to the allowed excursions from collinearity $[52,83]$. The $e Z$ e resonances, in contrast, exhibit much larger decay rates, which is not surprising in the light of the fully chaotic classical phase space of this configuration [18]. Yet, while doubly excited states decay, they may be long-lived enough and their density may be such that they play an important role in the internal structure of the atom, leaving strong signatures in scattering and photoionization signals $[3,5,32,84]$.

While the nonperturbative Coulomb coupling invalidates a straightforward classification of the resonance states of helium in terms of the independent-electron picture, these resonances remain organized in well-identifiable series that converge to the different single-ionization thresholds, for low excitation (see Fig. 2). In 1D Zee 
helium, the bound states below the first single-ionization threshold and the low-lying resonances may be labelled by approximate quantum numbers $(N, n)$, where $N$ denotes the series converging to the $N$-th single-ionization threshold, and $n$ corresponds to the degree of excitation within that series. While the electrons are indistinguishable, $N$ and $n$ may be seen as describing the state of the inner and the outer electron, respectively, in analogy with the uncoupled case $(\gamma=0)$. This description is expected to be valid at least up to $N=20$, where series start to overlap [85]. For $e Z e$ also the symmetry, even or odd, must be stated in addition to the approximate quantum number $(N, n)$.

\subsection{Complex rotation}

Analyzing the formation of autoionizing resonance states in helium beyond the qualitative picture given above is challenging for two reasons. First, the strong Coulomb coupling of the electrons excludes perturbative approaches which would be based on the independent-electron picture. For instance, while standard FeynmanDyson perturbation theory formally allows an exact description of the continuum coupling, the complex internal structure of the atom depicted above complicates the identification of a relevant subset of diagrams in most situations (see e.g. Ref. [86] for a discussion in the framework of nonequilibrium Green functions). Second, resonance states are not stationary, and their representation on a basis of stationary states of either the uncoupled $(\gamma=0)$ or the coupled Hamiltonian $(\gamma>0)$ necessarily involves a continuum of states [4]. The technique of complex rotation [47] circumvents both of these difficulties, as it allows the description of resonance states with bound-state techniques and numerical calculations with sets of integrable functions [87], as outlined below.

The foundations and the applications of the complex-rotation method have been reviewed e.g. in Refs. [41, 43-45, 47, 49]. In brief, Hamiltonian $H$ is replaced by

$$
H_{\theta}=R(\theta) H R(-\theta) \text {, }
$$

where $\theta$ is a real parameter, and $R(\theta)$ is the complex-rotation operator defined by

$$
R(\theta)=\exp \left(-\theta \frac{\boldsymbol{r} \cdot \boldsymbol{p}+\boldsymbol{p} \cdot \boldsymbol{r}}{2}\right),
$$

where $\boldsymbol{r}$ and $\boldsymbol{p}$ are position and momentum operators for a $d$-dimensional configuration space. While the parameter of the complex-rotation operator $R$ itself may be positive or negative, the $\theta$ parameter entering equation (2) is typically taken as a small positive angle for resonance poles belonging to the lower half complex plane to appear in the resolvent of the operator $H_{\theta}$ (see below). Practically, the rotated Hamiltonian $H(\theta)$ is obtained from $H$ through the substitution of $\boldsymbol{r}$ and $\boldsymbol{p}$ by $\boldsymbol{r}_{\theta}=R(\theta) \boldsymbol{r} R(-\theta)=\boldsymbol{r} e^{\mathrm{i} \theta}$ and $\boldsymbol{p}_{\theta}=R(\theta) \boldsymbol{p} R(-\theta)=\boldsymbol{p} e^{-\mathrm{i} \theta}$. In particular, this complex scaling of coordinates turns Hamiltonian (1) into

$$
H_{\theta}=-\frac{1}{2} e^{-2 \mathrm{i} \theta}\left(\frac{\partial^{2}}{\partial z_{1}^{2}}+\frac{\partial^{2}}{\partial z_{2}^{2}}\right)+e^{-\mathrm{i} \theta}\left(-\frac{Z}{z_{1}}-\frac{Z}{z_{2}}+\frac{\gamma}{\left|z_{2} \mp z_{1}\right|}\right) .
$$

For real, nonzero $\theta$, the operator $H_{\theta}$ is no longer Hermitian on the $L^{2}$ Hermitian domain of $H$, but becomes complex symmetric: $H_{\theta}^{\dagger}=\overline{H_{\theta}}=H_{-\theta}$, where the bar denotes complex conjugation. As $H_{\theta}$ is not Hermitian, its eigenvalues are not restricted to the real axis, and the eigenvectors will in general not be their own biorthogonal 
adjoints [88]. Because of the complex symmetry, however, the eigenvectors $\left|\psi_{i}^{\theta}\right\rangle$ of $H_{\theta}$ satisfy the following biorthogonality relation:

$$
\left\langle\overline{\psi_{i}^{\theta}} \mid \psi_{j}^{\theta}\right\rangle=\int \mathrm{d} \boldsymbol{r} \psi_{i}^{\theta}(\boldsymbol{r}) \psi_{j}^{\theta}(\boldsymbol{r})=\delta_{i j}
$$

where $\langle. \mid$.$\rangle is the usual Hermitian product, the bar again denotes complex conjugation,$ and the Kronecker delta may be replaced by a delta distribution for continuum states.

The spectrum of $H$ is defined as the set of complex numbers $z$ for which $H$ admits no bounded resolvent operator (Green's function) $G(z)=(z-H)^{-1}$ [89]. As outlined in section 2.1, this spectrum contains distinct proper eigenvalues, which show up as isolated poles in the resolvent $G(z)$ and correspond to bound states of the atom, as well as continua of improper eigenvalues, which lead to branch cuts in $G(z)$ and correspond to scattering states. Under complex rotation, this spectrum transforms as follows:

(i) The proper eigenvalues of $H_{\theta}$ coincide with those of $H$.

(ii) The continua of $H_{\theta}$ correspond to the continua of $H$, but are rotated about the ionization thresholds downwards $(\theta>0)$ into the complex plane by an angle of $2 \theta$.

(iii) For sufficiently large $\theta$, isolated complex eigenvalues $E_{i}^{\theta}=E_{i}-\mathrm{i} \Gamma_{i} / 2$ appear in the lower complex half plane $\left(\Gamma_{i}>0\right)$. In the limit of narrow and isolated resonances [90], these eigenvalues correspond to individual resonance states of $H$ with position $E_{i}$ and decay rate $\Gamma_{i}$, as discussed below.

The eigenstates $\left|\psi_{i}^{\theta}\right\rangle$ pertaining to the isolated complex eigenvalues of $H_{\theta}$ are squareintegrable. The states $\left|\psi_{i}\right\rangle=R(-\theta)\left|\psi_{i}^{\theta}\right\rangle$ obtained upon back-rotation are eigenstates of $H$, with the same complex eigenvalue $E_{i}^{\theta}$, and they decay under the action of $U(t)=\exp (-\mathrm{i} H t)$ at rate $\Gamma_{i}$. The resonance wave functions associated with these back-rotated states typically diverge exponentially as a function of distance, and thus lie outside the Hermitian domain of $H$. As a matter of fact, they may be found by imposing purely outgoing boundary conditions on the differential operator $H$ [90], but such an approach may prove difficult in practice [45]. In the setting of complex rotation, on the other hand, the square-integrable rotated resonance states $\left|\psi_{i}^{\theta}\right\rangle$ can be accurately calculated on a large but finite $L^{2}$ basis set. Let us now examine how these states may be used to describe the correlated electron dynamics.

\subsection{Complex-rotated states and real atomic dynamics}

While the eigenstates of $H_{\theta}$ themselves are unphysical, they provide a useful representation to describe the evolution of physical states under the action of the Hamiltonian $H$. Under the assumption that their set is complete, the eigenstates $\left|\psi_{i}^{\theta}\right\rangle$ of $H_{\theta}$ yield the resolution of identity

$$
\sum_{i}\left|\psi_{i}^{\theta}\right\rangle\left\langle\overline{\psi_{i}^{\theta}}\right|=\mathbb{1}
$$

where the sum runs over the bound states, the $L^{2}$ resonance states and the continuum states of $H_{\theta}$. The time evolution of a square integrable state $|\phi(t)\rangle=U(t)|\phi(0)\rangle$ and its overlap with a localized time-independent reference state $\left|\phi_{0}\right\rangle$ are then given by $[48,91]$

$$
\begin{aligned}
\left\langle\phi_{0} \mid \phi(t)\right\rangle & =\left\langle\phi_{0}\left|R(-\theta) e^{-\mathrm{i} H_{\theta} t} R(\theta)\right| \phi(0)\right\rangle \\
& =\sum_{i} e^{-\mathrm{i} E_{i}^{\theta} t}\left\langle\phi_{0}|R(-\theta)| \psi_{i}^{\theta}\right\rangle\left\langle\overline{\psi_{i}^{\theta}}|R(\theta)| \phi(0)\right\rangle .
\end{aligned}
$$


The initial state $|\phi(0)\rangle$ may be a nonstationary state of $H$ obtained e.g. by applying an external field to the atom in its ground state. When $|\phi(0)\rangle$ overlaps mostly with a single (back-rotated) resonance wave function $\left|\psi_{i_{0}}\right\rangle=R(-\theta)\left|\psi_{i_{0}}^{\theta}\right\rangle$, the dominant feature in the time evolution of $\left|\left\langle\phi_{0} \mid \phi(t)\right\rangle\right|^{2}$ is an exponential decay at rate $\Gamma_{i_{0}}=$ $-2 \operatorname{Im}\left(E_{i}^{\theta}\right)$ on intermediate time scales [40].

The above considerations can be developed in a more general setting and couched in the resolvent formalism. Indeed, identity (6) allows for a spectral representation of the Green's function of $H$ in terms of the eigenstates of $H_{\theta}$ [92]. More precisely, complex rotation directly provides the analytical continuation $G^{I I}(z)$ of $G(z)=$ $(z-H)^{-1}$ on the second Riemann sheet [49]‡:

$$
G^{I I}(z)=R(-\theta) \frac{1}{z-H_{\theta}} R(\theta)=\sum_{i} \frac{R(-\theta)\left|\psi_{i}^{\theta}\right\rangle \overline{\psi_{i}^{\theta}} \mid R(\theta)}{z-E_{i}^{\theta}} .
$$

This continuation is sound when expression (8) is applied on both sides on square integrable states, and $R(-\theta)$ and $R(\theta)$ are interpreted as acting to the left and to the right, respectively [49]. Expression (8) is free of branch cuts on the real axis. For real $E$ and $\theta>0$ (resp. $\theta<0$ ), $G^{I I}(E)$ thus coincides with the retarded (resp. advanced) Green's function $G^{ \pm}(E)=\lim _{\eta \rightarrow 0^{+}} G(E \pm \mathrm{i} \eta)$. Therefore, the difference between the retarded and advanced Green's functions may be cast into the form [49]

$$
G^{+}(E)-G^{-}(E)=\sum_{i}\left[\frac{R(-\theta)\left|\psi_{i}^{\theta}\right\rangle\left\langle\overline{\psi_{i}^{\theta}}\right| R(\theta)}{E-E_{i}^{\theta}}-\frac{R(\theta)\left|\overline{\psi_{i}^{\theta}}\right\rangle\left\langle\psi_{i}^{\theta}\right| R(-\theta)}{E-\overline{E_{i}^{\theta}}}\right],
$$

with $\theta>0$. Note that this expression also writes [93]

$$
G^{+}(E)-G^{-}(E)=-2 \pi \mathrm{i} \sum_{j} \rho_{j}(E)\left|\psi_{E, j}\right\rangle\left\langle\psi_{E, j}\right|,
$$

where the $\left|\psi_{E, j}\right\rangle$ denote the eigenstates of $H$ with energy $E$, the index $j$ labels other quantum numbers, and $\rho_{j}(E)$ is the associated density of states. As a consequence of expression (9), in particular, the local density of states or electronic density at energy $E$ is given by [49]

$$
\begin{aligned}
\rho(E, \boldsymbol{r}) & =-\frac{1}{2 \pi \mathrm{i}}\left[\left\langle\boldsymbol{r}\left|G^{+}(E)\right| \boldsymbol{r}\right\rangle-\left\langle\boldsymbol{r}\left|G^{-}(E)\right| \boldsymbol{r}\right\rangle\right] \\
& =-\frac{1}{\pi} \operatorname{Im}\left\langle\boldsymbol{r}\left|G^{+}(E)\right| \boldsymbol{r}\right\rangle=\frac{1}{\pi} \operatorname{Im} \sum_{i} \frac{\left\langle\boldsymbol{r}|R(-\theta)| \psi_{i}^{\theta}\right\rangle^{2}}{E_{i}^{\theta}-E} .
\end{aligned}
$$

Unlike expression (10), formulas (9) and (11) manifestly reflect the contributions of resonance states (bound states diluted in a continuum) to the density of states. In the framework of complex rotation, these contributions may be calculated accurately with bound-state techniques. In the case where the sum (11) is restricted to the contribution of one isolated resonance pole $E_{i_{0}}^{\theta}\left(\operatorname{Im} E_{i_{0}}^{\theta}<0\right)$, the associated electronic density reads

$$
\rho_{i_{0}}(\boldsymbol{r})=\int \frac{\mathrm{d} E}{\pi} \operatorname{Im} \frac{\left\langle\boldsymbol{r}|R(-\theta)| \psi_{i_{0}}^{\theta}\right\rangle^{2}}{E_{i_{0}}^{\theta}-E}=\operatorname{Re}\left[\left\langle\boldsymbol{r}|R(-\theta)| \psi_{i_{0}}^{\theta}\right\rangle^{2}\right],
$$

which typically diverges exponentially in space, as remarked above. The fact that $\rho_{i_{0}}(\boldsymbol{r})$ may assume negative values locally can be traced back to the single-pole

$\ddagger$ See also Ref. [93] for a general discussion of resonance poles and analytic continuation without the framework complex rotation. 
approximation, as the sum over all states of equation (11) would restore the positivity of the density [49]. Note also that the difference between $\rho_{i_{0}}(\boldsymbol{r})=\operatorname{Re}\left[\psi_{i_{0}}(\boldsymbol{r})^{2}\right]=$ $\operatorname{Re}\left[\left\langle\boldsymbol{r} \mid \psi_{i_{0}}\right\rangle\left\langle\overline{\psi_{i_{0}}} \mid \boldsymbol{r}\right\rangle\right]$ and $\left|\left\langle\boldsymbol{r}|R(-\theta)| \psi_{i_{0}}^{\theta}\right\rangle\right|^{2}=\left|\psi_{i_{0}}(\boldsymbol{r})\right|^{2}=\left\langle\boldsymbol{r} \mid \psi_{i_{0}}\right\rangle\left\langle\psi_{i_{0}} \mid \boldsymbol{r}\right\rangle$ arises from two ways of envisioning the single-pole approximation. The first one involves a coupling from e.g. a stationary state to the continuum at (real) energy $E$ close to $E_{i_{0}}$, with a density of states given by equation (9), while the second one assumes that the physical state is approximated by the back-rotated resonance wavefunction $\psi_{i_{0}}$ from the outset.

Let us now revisit the situation described below equation (7) and consider the perturbation of Hamiltonian $H$ by an additional term $V^{\prime}$, so that the total Hamiltonian reads $H^{\prime}=H+V^{\prime}$. Then, the transition amplitudes between stationary states $\left|\phi_{i n}\right\rangle$ and $\left|\phi_{f}\right\rangle$ of $H$ in the interaction picture are entirely determined by the elements of the $T$-matrix [93],

$$
\left\langle\phi_{f}|T(E)| \phi_{i n}\right\rangle=\left\langle\phi_{f}\left|V^{\prime}+V^{\prime} G^{\prime+}(E) V^{\prime}\right| \phi_{i n}\right\rangle,
$$

where $G^{\prime+}$ is the retarded Green's function of the total Hamiltonian $H^{\prime}$.In the limit of infinite perturbation times $\left|\phi_{i n}\right\rangle$ couples only to states $\left|\phi_{f}\right\rangle$ at energy $E_{f}=E_{i n}$, and the transition rate between $\left|\phi_{i n}\right\rangle$ and a continuum of states $\left|\psi_{E_{i n}, j}\right\rangle$ at this energy is

$$
\begin{aligned}
\gamma & =2 \pi \rho\left(E_{i n}\right) \sum_{j}\left|\left\langle\psi_{E_{i n}, j}\left|T\left(E_{i n}\right)\right| \phi_{i n}\right\rangle\right|^{2} \\
& =\mathrm{i}\left\langle\phi_{i n}\left|T^{\dagger}\left(E_{i n}\right)\left[G^{+}\left(E_{i n}\right)-G^{-}\left(E_{i n}\right)\right] T\left(E_{i n}\right)\right| \phi_{i n}\right\rangle .
\end{aligned}
$$

In the Born approximation, $T(E)$ reduces to $V^{\prime}$. Then, with the help of equation (9), the transition rate writes

$$
\gamma=2 \operatorname{Im} \sum_{i} \frac{\left\langle\overline{\psi_{i}^{\theta}}\left|R(\theta) V^{\prime}\right| \phi_{i n}\right\rangle^{2}}{E_{i}^{\theta}-E_{\text {in }}}=2 \operatorname{Im} \sum_{i} \frac{\left\langle\phi_{i n}\left|V^{\prime} R(-\theta)\right| \psi_{i}^{\theta}\right\rangle^{2}}{E_{i}^{\theta}-E_{\text {in }}} .
$$

As above, the initial state $\left|\phi_{i n}\right\rangle$ may be the ground state $|g\rangle$ of the atom in the presence of a laser field with frequency $\omega$. In that case $H^{\prime}$ involves the timedependent perturbation $V^{\prime}(t)=-\left(\boldsymbol{D}^{+}+\boldsymbol{D}^{-}\right) \cdot\left(\mathcal{E}^{+} e^{-\mathrm{i} \omega t}+\mathcal{E}^{-} e^{\mathrm{i} \omega t}\right)$ in the dipole approximation, with $\boldsymbol{D}^{ \pm}$the dipole transition operators and $\mathcal{E}^{ \pm}$the classical field amplitudes [94]. Then, upon substitution of $V^{\prime}$ by $-\boldsymbol{D}^{+} \mathcal{E}^{+}$and of $E_{\text {in }}$ by $E_{g}+\omega$, expression (15) directly gives the photoionization cross-section $\sigma(\omega)$, at leading order in the field intensity $[49,95]$. If $V^{\prime}\left|\phi_{i n}\right\rangle$ overlaps mostly with the back-rotated vector $R(-\theta)\left|\psi_{i_{0}}^{\theta}\right\rangle$ pertaining to an autoionizing resonance and $E_{i n}$ is chosen such that $\left|E_{\text {in }}-\operatorname{Re}\left(E_{i_{0}}^{\theta}\right)\right| \ll\left|\operatorname{Im}\left(E_{i_{0}}^{\theta}\right)\right|$, expression (15) may be approximated by

$$
\left.\gamma \simeq \frac{4}{\Gamma_{i_{0}}} \operatorname{Re}\left(\overline{\left\langle\psi_{i_{0}}^{\theta}\right.}\left|R(\theta) V^{\prime}\right| \phi_{i n}\right\rangle^{2}\right)
$$

where $\Gamma_{i_{0}}=-2 \operatorname{Im}\left(E_{i_{0}}^{\theta}\right)$ is the decay rate of the resonance state. The fact that $\gamma$ is inversely proportional to $\Gamma_{i_{0}}$ is well known from resonance-mediated decay [93], and reflects that in the above example the photoionization of $\left|\phi_{i n}\right\rangle$ is mediated by the autoionizing resonance. Most importantly, the back-rotated resonance wave function $\psi_{i_{0}}(\boldsymbol{r})=\left\langle\boldsymbol{r}|R(-\theta)| \psi_{i_{0}}^{\theta}\right\rangle$ determines not only the strength of the coupling between the initial state and the resonance state, but also how the initial state decays into continuum states. Indeed, as emphasized in the following sections, the resonance wave function carries in itself the final (continuum) states of the decay process.

The above discussion illustrates that while the exact ionization dynamics of an atomic state generally involves a continuum of eigenstates of $H_{\theta}$, the most significant part of it may often be captured in a single resonance state $\left|\psi_{i_{0}}^{\theta}\right\rangle$ under suitable 
conditions. The decay processes of such individual resonance states is the subject of the remainder of this paper. We emphasize that, rather than describing the transition from a stationary state to the continuum in the vicinity of a resonance, as illustrated above, we shall concentrate for simplicity on the assumption that the physical state under scrutiny is described from the outset by the back-rotated wavefunction of that resonance.

\subsection{Energies and wave functions of atomic resonances in $1 D$ helium}

We use a Sturmian basis to compute the eigenvalues and eigenvectors of the rotated Hamiltonian (4) efficiently with standard techniques for sparse matrix eigenvalue problems [50, 83, 85, 96, 97].

Here the Sturmian functions are defined by [85]

$$
S_{n}^{(\alpha)}(r)=\frac{1}{\sqrt{n}}(-1)^{n} \frac{2 r}{\alpha} e^{-r / \alpha} L_{n-1}^{(1)}\left(\frac{2 r}{\alpha}\right),
$$

where $L_{n}^{(1)}$ denotes an associated Laguerre polynomial of order $n$ [98], and $\alpha>0$ is introduced as a scaling parameter. The Sturmian functions $\left\{S_{n}^{(\alpha)}(r), n \geq 1\right\}$ form a complete orthonormal set with respect to the weight function $1 / r$ on the interval $I=[0,+\infty[$ (for a detailed discussion of completeness properties, see e.g. Ref. [99]). In particular, it is worth noting that $S_{N}^{(N / Z)}(r)$ coincides up to a normalization factor with the wave function of the $N$ th $1 \mathrm{D}$ hydrogenic bound state in the Coulomb potential $-Z / r$. This implies that for a specific scaling $\alpha$ one such hydrogenic state may be represented faithfully by a single element of the Sturmian basis. While the complete (yet discrete) Sturmian basis is able to represent all bound and continuum states of the hydrogen or helium atom, in any practical computation the basis must be truncated to contain only functions below a certain $N_{\max }$. This truncation introduces a soft finitesize cut-off at a typical distance of $2 \alpha N_{\max }$ Bohr radii. This distance corresponds to the approximate position of the outermost extremum of $S_{N_{\max }}^{(\alpha)}(r)$, as given by expression (17). Because of this cut-off, the atomic continua are discretized into quasi-continua, Rydberg series are resolved only up to a certain degree of excitation, and the calculated ionization thresholds are shifted slightly downwards with respect to the exact values (see Refs. [83, 100] and below). By increasing $N_{\max }$ and adapting $\alpha$, however, these effects can be made small.

Using a Sturmian basis set has the following advantage from the computational point of view: The operators

$$
\begin{aligned}
S_{ \pm}^{(\alpha)} & =\frac{r}{2 \alpha}+\frac{\alpha r}{2} \frac{\mathrm{d}^{2}}{\mathrm{~d} r^{2}} \mp r \frac{\mathrm{d}}{\mathrm{d} r} \\
S_{3}^{(\alpha)} & =\frac{r}{2 \alpha}-\frac{\alpha r}{2} \frac{\mathrm{d}^{2}}{\mathrm{~d} r^{2}}
\end{aligned}
$$

form a Lie algebra of ladder operators with the commutators $\left[S_{-}^{(\alpha)}, S_{+}^{(\alpha)}\right]=2 S_{3}^{(\alpha)}$ and $\left[S_{3}^{(\alpha)}, S_{ \pm}^{(\alpha)}\right]= \pm S_{ \pm}^{(\alpha)}$, and the following action on the Sturmian functions $S_{n}^{(\alpha)}(r)=$ $\left\langle r \mid S_{n}^{(\alpha)}\right\rangle$ of equation (17) [101, 102]:

$$
\begin{aligned}
S_{ \pm}^{(\alpha)}\left|S_{n}^{(\alpha)}\right\rangle & =\sqrt{n(n \pm 1)}\left|S_{n \pm 1}^{(\alpha)}\right\rangle \\
S_{3}^{(\alpha)}\left|S_{n}^{(\alpha)}\right\rangle & =n\left|S_{n}^{(\alpha)}\right\rangle .
\end{aligned}
$$


To exploit these properties we use product bases of two sets of Sturmian functions that are fitted to the specific configuration.

For the $e Z e$ configuration we expand $\psi^{\theta}\left(z_{1}, z_{2}\right)$ on a (anti-) symmetrized product basis as

$$
\begin{aligned}
\psi_{\text {odd }}^{\theta}\left(z_{1}, z_{2}\right) & =\sum_{n_{1}=1}^{N} \sum_{n_{2}=n_{1}+1}^{N} \frac{C_{n_{1} n_{2}}}{\sqrt{2}}\left(S_{n_{1}}^{(\alpha)}\left(z_{1}\right) S_{n_{2}}^{(\alpha)}\left(z_{2}\right)-S_{n_{1}}^{(\alpha)}\left(z_{2}\right) S_{n_{2}}^{(\alpha)}\left(z_{1}\right)\right), \\
\psi_{\text {even }}^{\theta}\left(z_{1}, z_{2}\right) & =\sum_{n_{1}=1}^{N} \sum_{n_{2}=n_{1}+1}^{N} \frac{C_{n_{1} n_{2}}}{\sqrt{2}}\left(S_{n_{1}}^{(\alpha)}\left(z_{1}\right) S_{n_{2}}^{(\alpha)}\left(z_{2}\right)+S_{n_{1}}^{(\alpha)}\left(z_{2}\right) S_{n_{2}}^{(\alpha)}\left(z_{1}\right)\right) \\
& +\sum_{n_{1}=1}^{N} C_{n_{1} n_{1}} S_{n_{1}}^{(\alpha)}\left(z_{1}\right) S_{n_{1}}^{(\alpha)}\left(z_{2}\right),
\end{aligned}
$$

for the odd and even states respectively. It is now our goal to express the operators in the eigenvalue equation as polynomials in the ladder operators. This is not immediately possible, because they admit no representation of $\frac{1}{r}$. To overcome this problem we multiply the eigenvalue equation from the left by a factor of $e^{3 i \theta} z_{1} z_{2}\left(z_{1}+z_{2}\right)$. Note that for $e Z e$ we have $\left|z_{1} \mp z_{2}\right|=\left(z_{1}+z_{2}\right)$. This leaves us with the generalized eigenvalue problem

$$
A_{\theta} \psi^{\theta}\left(z_{1}, z_{2}\right)=E^{\theta} B_{\theta} \psi^{\theta}\left(z_{1}, z_{2}\right),
$$

with

$$
\begin{aligned}
& A_{\theta}=e^{3 \mathrm{i} \theta} z_{1} z_{2}\left(z_{1}+z_{2}\right) H_{\theta} \\
& B_{\theta}=e^{3 \mathrm{i} \theta} z_{1} z_{2}\left(z_{1}+z_{2}\right) .
\end{aligned}
$$

Consequently all the fractions appearing in the Hamiltonian cancel. Then, with the help of expressions (18) and (19), the operators $A_{\theta}$ and $B_{\theta}$ in equations (26) and (27) are written as polynomials of degree three in $S_{ \pm}^{\left(\alpha_{i}\right)}$ and $S_{3}^{\left(\alpha_{i}\right)}$. The small degree of these polynomials enforces strong selection rules between the elements of the product basis. As a result, the matrix representations of the operators of equation (25) in the Sturmian basis are very sparse, and the eigenvalue problem (25) is amenable to standard numerical techniques based on Krylov subspaces [103].

In Zee helium we exploit the stronger symmetry that was discussed in Section 2 in a different way. Owing to the exchange symmetry and the singularity of the Coulomb potential between electrons, the two-electron Zee wave function separates into

$$
\psi\left(z_{1}, z_{2}\right)=\psi_{>}\left(z_{1}, z_{2}\right) \pm \psi_{>}\left(z_{2}, z_{1}\right),
$$

where $\psi_{>}\left(z_{1}, z_{2}\right)$ is defined on the domain $0<z_{2}<z_{1}$, and vanishes at its boundaries. On this domain, upon introduction of the perimetric coordinates

$$
\begin{aligned}
& x=z_{1}-z_{2} \\
& y=z_{2},
\end{aligned}
$$

one is left with the eigenvalue problem

$$
H_{\theta} \phi_{>}^{\theta}(x, y)=E^{\theta} \phi_{>}^{\theta}(x, y),
$$

with $\phi_{>}^{\theta}(x, y)=\psi_{>}^{\theta}(x+y, y)$ and

$H_{\theta}=e^{-2 \mathrm{i} \theta}\left(-\frac{\partial^{2}}{\partial x^{2}}-\frac{1}{2} \frac{\partial^{2}}{\partial y^{2}}+\frac{\partial^{2}}{\partial x \partial y}\right)+e^{-\mathrm{i} \theta}\left(-\frac{Z}{x+y}-\frac{Z}{y}+\frac{1}{x}\right)$. 
Following Ref. [83], equation (31) is replaced by the generalized eigenvalue problem

$$
A_{\theta} \phi^{\theta}(x, y)=E^{\theta} B_{\theta} \phi^{\theta}(x, y),
$$

where

$$
\begin{aligned}
& A_{\theta}=e^{4 \mathrm{i} \theta} x y(x+y) H_{\theta}(x+y) \\
& B_{\theta}=e^{4 \mathrm{i} \theta} x y(x+y)^{2},
\end{aligned}
$$

which is obtained upon multiplication of equation (31) by $e^{3 i \theta} x y(x+y)$ from the left, with the substitution

$$
\phi_{>}^{\theta}(x, y)=e^{\mathrm{i} \theta}(x+y) \phi^{\theta}(x, y) .
$$

Now $\phi^{\theta}(x, y)$ is expanded on a Sturmian product basis as

$$
\phi^{\theta}(x, y)=\sum_{n_{x}=1}^{N_{x}} \sum_{n_{y}=1}^{N_{y}} C_{n_{x} n_{y}} S_{n_{x}}^{\left(\alpha_{x}\right)}(x) S_{n_{y}}^{\left(\alpha_{y}\right)}(y),
$$

where $N_{x}$ and $N_{y}$ arise from the truncation of the basis in any practical computation, and $\alpha_{x}$ and $\alpha_{y}$ are scaling factors of the basis in the $x$ and $y$ directions. The latter can be adapted independently for an optimal representation of certain electronic configurations in the truncated basis. In this respect, note that multiplying $H_{\theta}$ from the left by $e^{3 i \theta} x y(x+y)$, without additionnal $e^{\mathrm{i} \theta}(x+y)$ factor from the right, actually suffices for a polynomial representation of the resulting operator in terms of the ladder operators $S_{ \pm}^{\left(\alpha_{i}\right)}$ and $S_{3}^{\left(\alpha_{i}\right)}$. In that case, the total degree of the polynomial is only three and the selection rules are even stronger (the operators $A_{\theta}^{\prime}=e^{3 i \theta} x y(x+y) H_{\theta}$ and $B_{\theta}^{\prime}=e^{3 \mathrm{i} \theta} x y(x+y)$ of the resulting eigenvalue problem couple each element of the Sturmian basis to a maximum of 20 other elements, with $\left|\Delta n_{x}\right| \leq 2$ and $\left|\Delta n_{y}\right| \leq 2$ ). Note however, that the matrix representation of $A_{\theta}^{\prime}$ in the Sturmian basis is not complex-symmetric, which limits the efficiency of suitable eigenvalue routines.

In this way we have described both $1 \mathrm{D}$ configurations as sparse eigenvalue problems that are accessible for standard numerical techniques. For the calculation of the eigenvalues and eigenvectors, we used the SLEPc library [103], which itself is build on top of PETSc [104, 105], in combination with the parallel solver MUMPS [106, 107].

Figure 2 shows a complex-rotated Zee spectrum obtained via the Krylov-Schur method as described in Ref. [108]. This spectrum displays the features discussed in section 2.2, in particular the isolated eigenvalues $E_{i}^{\theta}=\operatorname{Re}\left(E_{i}^{\theta}\right)-\mathrm{i} \Gamma_{i} / 2$ in the lower half of the complex plane $\left(\Gamma_{i}>0\right)$, which correspond to resonance states (see bottom right panel). Figure 3 shows a similar spectrum for the $e Z e$ case. The two series associated with even and odd symmetry, respectively, can be clearly distinguished.

For the further analysis with regards to partial rates, we need to calculate wave functions from the now available eigenvectors. To this end we exploit the second essential advantage brought along by the Sturmian basis set, namely that the matrix elements of the complex-rotation operator (3) between Sturmians are known analytically [101, 102, 109]:

$$
\begin{aligned}
&\left\langle\widetilde{S}_{n}^{(\alpha)}|R(-\theta)| S_{n^{\prime}}^{(\alpha)}\right\rangle=e^{\mathrm{i} \theta / 2} \mathrm{i}^{n-n^{\prime}} \sqrt{n n^{\prime}}\left(\cos \frac{\theta}{2}\right)^{-\left(n+n^{\prime}\right)}\left(\sin \frac{\theta}{2}\right)^{n+n^{\prime}-2} \\
& \times{ }_{2} F_{1}\left[-n+1,-n^{\prime}+1 ; 2 ;\left(\sin \frac{\theta}{2}\right)^{-2}\right],
\end{aligned}
$$



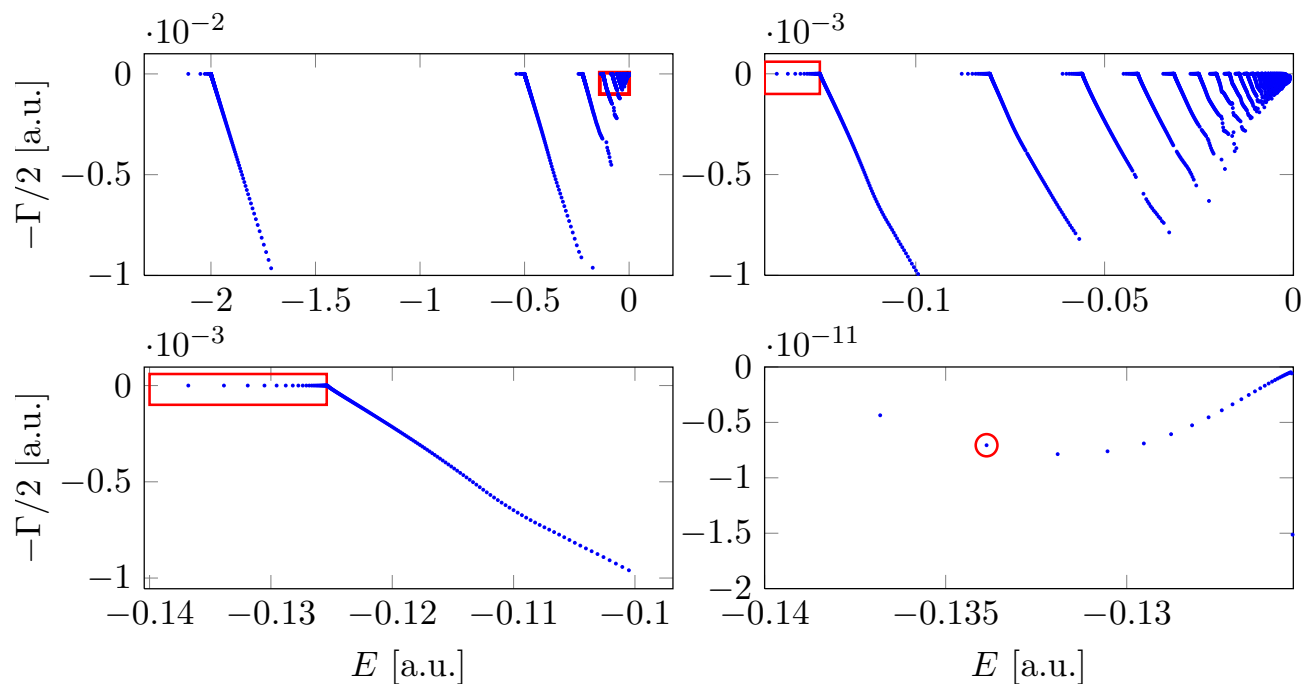

Figure 2. (Colour online) Spectrum of $1 \mathrm{D}$ helium in the Zee configuration under complex rotation. The eigenvalues in blue are obtained from the solution of the eigenvalue problem (33) in the Sturmian basis through the Krylov-Schur method, with numerical parameters $\theta=0.005, \alpha_{x}=50, \alpha_{y}=50, N_{x}=300$, $N_{y}=150$. The top left panel gives an overview of the spectrum with the ground state at -2.108 a.u.and the double-ionization threshold (DIT) at zero energy. The following panels (from left to right, and top to bottom) are consecutive zooms into the marked areas. The different series corresponding to states of the inner electron are visible with their rotated (and discretized) continua (see sections 2.2 and 2.4). The bound states below the first single-ionization threshold at $E_{1}^{\mathrm{SIT}}=-2$ a.u. lie on the real axis, whereas the resonances above $E_{1}^{\text {SIT }}$ have a small imaginary part, as shown by the bottom right panel. The two last panels (bottom) show the $N=4$ series, i.e. the series corresponding to the third excited state of the inner electron. The bottom right graph highlights the $(4,6)$ resonance with a red circle. This resonance has the eigenvalue $E_{(4,6)}=\left(-1.3387 \cdot 10^{-1}-7.06 \cdot 10^{-12} \mathrm{i}\right)$ a.u., i.e. an energy of -0.13387 a.u. and a decay rate of $1.41 \cdot 10^{-11}$ a.u.

where we use the notation $\langle\widetilde{\phi} \mid \psi\rangle=\int_{0}^{+\infty} r^{-1} \overline{\phi(r)} \psi(r) \mathrm{d} r$, and ${ }_{2} F_{1}$ is the hypergeometric function [98].

The exact calculation again differs for the two configurations. For the Zee case the representation of $R(-\theta)\left|\phi_{i}^{\theta}\right\rangle$ in the Sturmian basis gives the back-rotated resonance wave function $\phi_{>, i}(x, y)=\left\langle x, y|R(-\theta)| \phi_{>, i}^{\theta}\right\rangle=\left\langle x, y\left|R(-\theta) e^{\mathrm{i} \theta}(x+y)\right| \phi_{i}^{\theta}\right\rangle=$ $(x+y)\left\langle x, y|R(-\theta)| \phi_{i}^{\theta}\right\rangle$ on the perimetric domain without analytic continuation, by evaluating the Sturmian functions with real arguments $x$ and $y$. The resulting spatial wave function $\psi_{>, i}\left(z_{1}, z_{2}\right)=\phi_{>, i}(x, y)$ is extended to the full Zee domain by simple symmetrization, which yields $\psi_{i}\left(z_{1}, z_{2}\right)$. Figure 4 shows the electronic density $\left|\psi\left(z_{1}, z_{2}\right)\right|^{2}$ obtained upon back-rotation of the $(4,6)$ resonance state (marked by a red circle in Fig. 2) on the entire $\left(z_{1}, z_{2}\right)$ domain. The density plot (on a logarithmic scale) has two important features: a central part that extends up to $100-200$ a.u. in $z_{1}$ direction and an asymptotic contribution that runs along the $z_{1}$ axis beyond 250 a.u. The central part corresponds to the remnant of a bound state of the two electrons around the nucleus. Counting the nodal lines along both directions confirms the approximate quantum numbers. At distance $z_{1}=100$ a.u., for instance, Fig. 4 


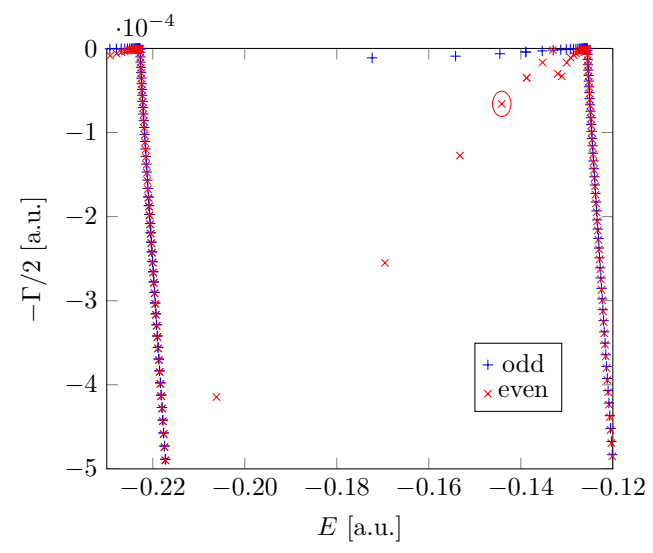

Figure 3. (Colour online) Spectrum of $1 \mathrm{D}$ helium in the eZe configuration under complex rotation. This part of the spectrum shows the $N=4$ series of resonances between the $N=3$ continuum on the left and the $N=4$ continuum on the right. The blue + symbols refer to the odd states, the red $\mathrm{x}$ symbols to those with even symmetry (see discussion in section 2). While there is almost a one-to-one correspondence between the two symmetries for the resonances, the even states exhibit much higher decay rates. Furthermore, since the $(4,4)$ state is only possible in the even symmetry, this series has one more state. This is also the reason for the deviation from the smooth behaviour of the resonances approaching the threshold: Around $E=-0.13$ a.u. we see the intrusion of a perturber from the $N=5$ series in the even configuration. In the odd symmetry this only happens in even higher series. The red circle marks the $(4,7, \mathrm{e})$ resonance. It will be analysed in more detail below, and has an eigenvalue of $E_{(4,7, e)}=\left(-1.4415 \cdot 10^{-1}-6.5915 \cdot 10^{-5}\right.$ i) a.u., i.e. an energy of -0.14415 a.u. and a decay rate of $1.3183 \cdot 10^{-4}$ a.u.

reveals three nodes in $z_{2}$ direction, in agreement with $N=4$. A closer inspection of the region around the origin (not shown here) also confirms that the central part displays four nodes in $z_{1}$ direction, close to the $z_{1}$ axis. The asymptotic part, on the other hand, corresponds to the unbound motion of one of the electrons, and signals the admixture of singly ionized states. Interestingly, the asymptotic density profile exhibits a clear nodal structure along the $z_{2}$ direction, as in the bound part. In the transition regime between 200 a.u. and 250 a.u. one nodal line vanishes. This indicates that in the final state of the autoionization process, the remaining electron has dropped to level three of the helium ion $\mathrm{He}^{+}$.

For the $e Z e$ case we start by transforming the coefficients in the (anti-) symmetrized basis into the ordinary product basis of Sturmians for $z_{1}$ and $z_{2}$. The evaluation of the spatial wave function is then done as in the Zee case, without the additional $(x+y)$ factor and immediately on the whole domain. The resulting electronic density $\left|\psi\left(z_{1}, z_{2}\right)\right|^{2}$ for the $(4,7$, even) state is depicted in Fig. 5. It shares many features with its Zee counterpart. There are, however, several differences: Since the state is even, there is no nodal line along the diagonal. Instead here we find an antinode, only interrupted by the nodes in $z_{1}$ or $z_{2}$ direction. The density also shows a central and an asymptotic part. The extension of the central part is considerably smaller, which is unsurprising, since the two electrons here are on opposite sides of the nucleus. As compared to Fig. 4, the asymptotic part, now starting around 100 a.u., has a much larger weight. This is a result of the far larger decay rates of the eZe 


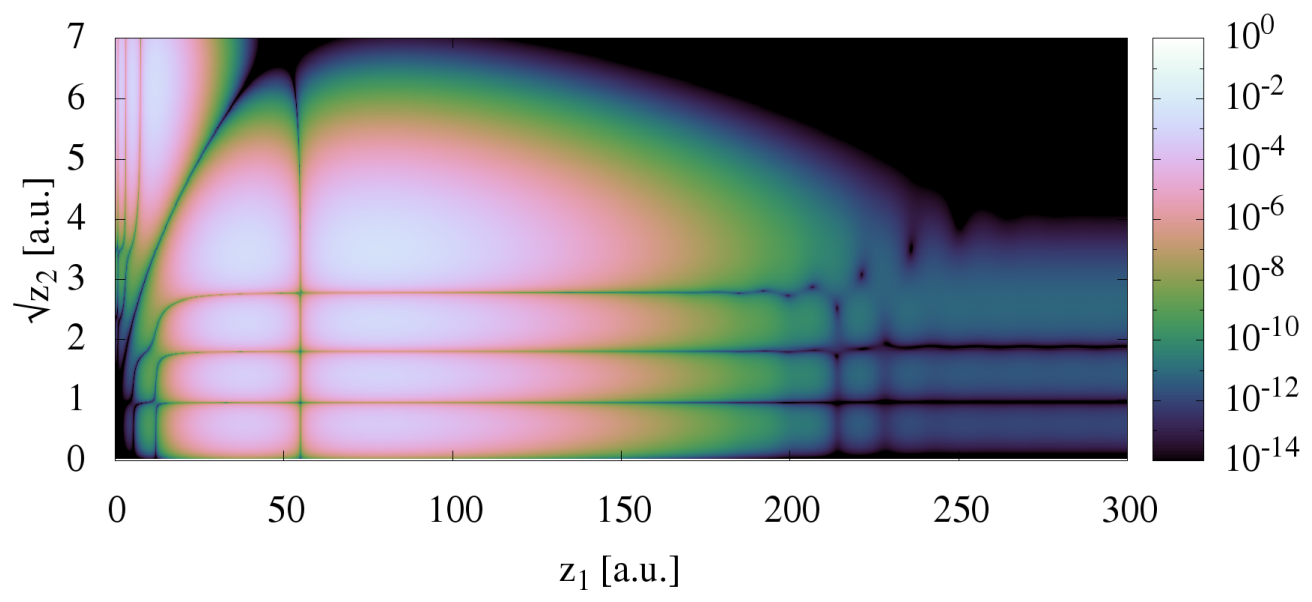

Figure 4. Electronic density $\left|\psi\left(z_{1}, z_{2}\right)\right|^{2}$ of the Zee $(4,6)$ resonance, as obtained upon back-rotation of the associated resonance wave function $\left|\phi_{>}^{\theta}\right\rangle$ (see section 2.4) and extension to the entire $\left(z_{1}, z_{2}\right)$ domain. The numerical parameters are $\theta=0.001, \alpha_{x}=2, \alpha_{y}=2, N_{x}=6000, N_{y}=300$. The $z_{2}$ direction is scaled with the square root to improve the visibility of the nodal structure. The clearly visible nodal line on the $z_{1}=z_{2}$ diagonal is distorted accordingly. The density profile reveals a central part, that quickly tails off exponentially after its maximum at around $z_{1}=100$ a.u., which corresponds to a bound state of the two electrons around the nucleus, and an asymptotic part along the $z_{1}$ axis $\left(z_{1} \gtrsim 250\right.$ a.u. $)$ which indicates the admixture of singly ionized states.

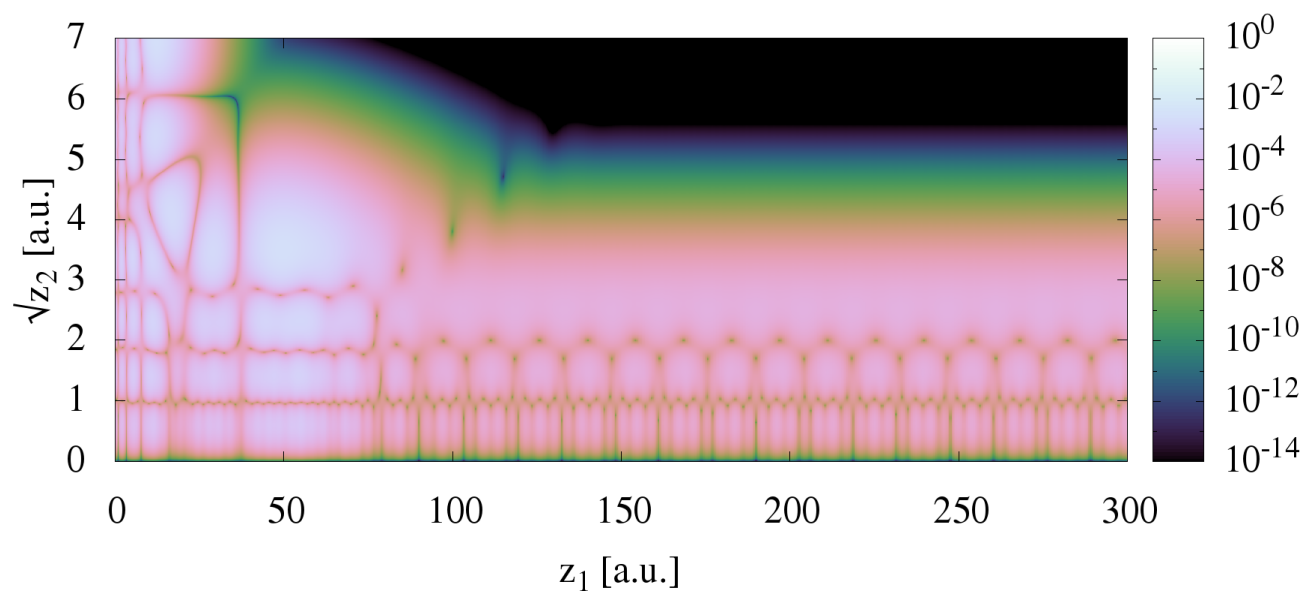

Figure 5. Electronic density $\left|\psi\left(z_{1}, z_{2}\right)\right|^{2}$ of the $e Z e(4,7, e)$ resonance, as obtained upon back-rotation of the associated resonance wave function $\left|\phi_{>}^{\theta}\right\rangle$ (see section 2.4). The numerical parameters are $\theta=0.005, \alpha=2, N=1500$. The $z_{2}$ direction is scaled with the square root to improve the visibility of the nodal structure. The density profile reveals a central part $\left(z_{1} \lesssim 100\right.$ a.u. $)$ which corresponds to a bound state of the two electrons around the nucleus, and an asymptotic part along the $z_{1}$ axis $\left(z_{1} \gtrsim 150\right.$ a.u.) which indicates the admixture of singly ionized states. 
configuration. Additionally there are further variations or ripples in the asymptotic part. These are an effect of the presence of several open channels and will be examined more closely in Sect. 4.

The above examples demonstrate that in contrast to the complex-rotated spectrum, which only gives access to the total decay rate of individual resonance states, an inspection of the back-rotated resonance wave function may reveal how those resonance states decay. While the presented resonance wave functions lend themselves to a clear qualitative interpretation, the analysis of decaying resonance states can be put on a systematic and quantitative basis by defining channel wave functions and computing the associated partial decay rates. In the following section, we show how the computation of such partial decay rates can be achieved in the setting of complex rotation.

\section{Partial decay rates from complex dilation}

In this section we present a general method for the calculation of partial decay rates. First we recall the calculation of rates from configuration-space analysis of wave functions. Then we demonstrate how wave functions can be decomposed into partial wave functions in a way compatible with the calculation of rates. This allows for the calculation of partial rates.

\subsection{Total decay rates and flux densities}

A decay rate is the rate at which the probability of a state in some region of configuration space decreases. More specifically, in this case we are looking at bound continuum transitions. Therefore the rate we are interested in is the rate of loss of probability from the region of configuration space that is associated with an initial state, here a bound state.

First we describe the calculation of rates from wave functions in configuration space. The setup is as follows: We have a configuration space $C$, whose coordinates we write as $\boldsymbol{r}$. Furthermore we have a time dependent state $\left|\psi_{t}\right\rangle$. Due to local conservation of probability density (see e.g. [110]), we have the continuity equation

$$
\frac{\partial}{\partial t}\left|\psi_{t}(\boldsymbol{r})\right|^{2}=-\nabla \boldsymbol{j}_{t}(\boldsymbol{r}),
$$

where $\boldsymbol{j}$ is the probability current density.

We are interested in the decay of the state. Therefore we assume the existence of a reaction region, for example the spatial region where the electronic density is localized at the onset of an ionization process. Due to the long-range nature of the Coulomb potential, this region is here not bounded. We choose a compact volume $V_{R} \subset C$ that is centered on this reaction region and parameterized by a length scale $R$, such that $\lim _{R \rightarrow \infty} V_{R}=C$. The intuitive picture is the sphere of appropriate dimension and radius $R$. However we will see that other shapes can be advantageous.

By integration over $V_{R}$ and application of Gauss' theorem, the loss of probability density inside $V_{R}$ is given by

$$
\int_{V_{R}} \frac{\mathrm{d}}{\mathrm{d} t}\left|\psi_{t}\right|^{2} \mathrm{~d} V=-\oint_{\partial V_{R}} \boldsymbol{j}_{t} \mathrm{~d} \boldsymbol{S} .
$$

If the state exhibits a simple exponential decay with decay rate $\Gamma$ we know that

$$
\frac{\mathrm{d}}{\mathrm{d} t}\left|\psi_{t}\right|^{2}=-\Gamma\left|\psi_{t}\right|^{2},
$$


hence if we evaluate (40) for such a state we find

$$
\Gamma=\frac{\oint_{\partial V_{R}} \boldsymbol{j}_{t} \mathrm{~d} \boldsymbol{S}}{\int_{V_{R}}\left|\psi_{t}\right|^{2} \mathrm{~d} V},
$$

i.e. the ratio on the right hand side is constant and independent of $R$, even though both numerator and denominator might diverge for $R \rightarrow \infty$. The denominator, i.e. the probability density inside $V_{R}$, is readily integrated for a given $R$. Thus the remaining task is the calculation of flux of the probability current density $j$ through $\partial V_{R}$. Note that it is sufficient to calculate the component of $\boldsymbol{j}$ that is perpendicular to the boundary. To account for this we define $\tilde{j}:=\boldsymbol{j} \boldsymbol{n}$, where $\boldsymbol{n}$ is the surface normal, i.e. $\mathrm{d} \boldsymbol{S}=\boldsymbol{n} \mathrm{d} S$.

For a standard discussion of current densities in the quantum-mechanical setting we refer to the literature (e.g. [111]). Here we only recall the necessary results: The current density $\boldsymbol{j}_{t}(\boldsymbol{r})$ at point $\boldsymbol{r}$ and time $t$ is obtained as the expectation value of the current-density operator $\boldsymbol{J}(\boldsymbol{r})$, i.e.

$$
\begin{aligned}
& \boldsymbol{j}_{t}(\boldsymbol{r})=\left\langle\psi_{t}|\boldsymbol{J}(\boldsymbol{r})| \psi_{t}\right\rangle, \\
& \boldsymbol{J}(\boldsymbol{r})=\frac{1}{2}(\boldsymbol{p}|\boldsymbol{r}\rangle\langle\boldsymbol{r}|+| \boldsymbol{r}\rangle\langle\boldsymbol{r}| \boldsymbol{p}) .
\end{aligned}
$$

This naturally leads to the current-density operator in direction of the boundary surface normal

$$
\tilde{J}(\boldsymbol{r})=\frac{1}{2}(\tilde{p}|\boldsymbol{r}\rangle\langle\boldsymbol{r}|+| \boldsymbol{r}\rangle\langle\boldsymbol{r}| \tilde{p}) .
$$

In the context of back-rotated wave functions from complex rotation, $|\psi\rangle=$ $R(-\theta)\left|\psi_{i_{0}}^{\theta}\right\rangle$ is not normalizable. Yet, the associated probability density $\langle\boldsymbol{r} \mid \psi\rangle\langle\psi \mid \boldsymbol{r}\rangle=$ $|\psi(\boldsymbol{r})|^{2}$ is finite everywhere. Furthermore, as discussed in section 2.2, the back-rotated wave function is still a solution to the time-independent Schrödinger equation with eigenvalue given by the complex eigenvalue of the rotated wave function. With the usual time evolution it therefore also generates a solution of the time-dependent Schrödinger equation with a purely exponential decay. Hence the normal derivation of the current-density operator works as well as the calculation of the current density as its expectation value.

Thus the derivation is complete: Given a wave function in configuration space that exhibits a purely exponential decay we can calculate the decay rate $\Gamma$ by analyzing the probability current density as given by the expectation value of the current density operator according to (42). Since the decay rate is here assumed constant in time, we now drop the index $t$ and always imply $t=0$. Let us now decompose the wave function to access partial rates.

\subsection{Partial rates}

Partial rates are rates associated with particular decay channels. What constitutes a channel can vary with the problem at hand, but will usually be defined by some condition on a part of the system. It is thus nothing but a subspace in Hilbert space. We treat the various channels by the projectors onto these subspaces.

To show that this perspective leads to a useful concept of partial rates, let us assume that we have a family of projectors $P_{N}$, such that

$$
\begin{aligned}
\sum_{N} P_{N} & =\mathbb{1} \\
P_{N} P_{M} & =\delta_{N M} P_{N},
\end{aligned}
$$


i.e. the corresponding subspaces are orthogonal and span the Hilbert space.

The goal is now to split the right hand side of (42) into a sum of terms that can be connected with the decay into given channels. For this we keep the denominator and split the numerator according to the channels.

$$
\begin{aligned}
\oint_{\partial V_{R}} \boldsymbol{j}(\boldsymbol{r}) \mathrm{d} \boldsymbol{S}(\boldsymbol{r}) & =\oint_{\partial V_{R}}\langle\psi|\tilde{J}(\boldsymbol{r})| \psi\rangle \mathrm{d} S \\
& =\sum_{N} \oint_{\partial V_{R}}\left\langle\psi\left|P_{N}^{2} \tilde{J}(\boldsymbol{r})\right| \psi\right\rangle \mathrm{d} S \\
& =\sum_{N} \oint_{\partial V_{R}}\left\langle\psi\left|P_{N} \tilde{J}(\boldsymbol{r}) P_{N}\right| \psi\right\rangle \mathrm{d} S-\sum_{N} \oint_{\partial V_{R}}\left\langle\psi\left|P_{N}\left[\tilde{J}(\boldsymbol{r}), P_{N}\right]\right| \psi\right\rangle \mathrm{d} S(.50)
\end{aligned}
$$

The second term of this sum will vanish if we choose $V_{R}$ compatible with the channels. We will see what this means more clearly in the application of the method in section 4 .

We introduce the projected wave function $\left|\psi_{N}\right\rangle:=P_{N}|\psi\rangle$ and define partial rates $\Gamma_{N}(R)$ as

$$
\Gamma_{N}(R)=\frac{\oint_{\partial V_{R}}\left\langle\psi_{N}|\tilde{J}(\boldsymbol{r})| \psi_{N}\right\rangle \mathrm{d} S}{\int_{V_{R}}|\psi|^{2} \mathrm{~d} V} .
$$

This is not a straightforward application of (42) to the projected wave functions, since the denominator is in fact calculated with the full wave function. Still, if $V_{R}$ is chosen such that the second term in (50) vanishes, we see that the partial rates sum up to the total rate:

$$
\Gamma=\sum_{N} \Gamma_{N}(R)
$$

Note that these partial rates depend on the extension of $V_{R}$. Their sum, however, the total rate $\Gamma$, stays constant for all $R$. Furthermore this gives us a well defined way to evaluate the partial rates for any $R$, and thus enables access to the asymptotic behaviour, without any ad-hoc assumptions on the final states. In other words, we can calculate the involved quantities as functions of $R$ and identify the asymptotic region as the part where no more interplay among the channels is observed, directly from the data. This is in contrast to other approaches that assume a specific final state both for the expelled particle and the remaining system [54-56, 67, 74, 76, 112]. Since we want to study these quantities as functions of $R$, the following definitions will be useful:

$$
\begin{aligned}
& \gamma_{(N)}(R)=\oint_{\partial V_{R}}\left\langle\psi_{(N)}|\tilde{J}(\boldsymbol{r})| \psi_{(N)}\right\rangle \mathrm{d} S \\
& D(R)=\int_{V_{R}}|\psi|^{2} \mathrm{~d} V .
\end{aligned}
$$

It is at this point useful to recall that the back-rotated wave function of a resonance computed with complex rotation is a static representation of a dynamic process. Starting from an initial state that corresponds to a bound state, one or several decay processes take place that lead to a net outgoing flux of probability density. This, in turn, generates a spatially unbounded density distribution. In the case of an expelled particle, this gives the probability to find the particle in some (infinitesimal) space region. We have up to now neglected the time domain. To reconcile the two pictures, we recognize that time and space in the case of a moving classical particle with fixed energy are linked rather directly as $\Delta_{x}=v \Delta_{t}$, where $v$ is 
the velocity of the particle. In this sense a point in space outside the bound part of the wave function can be associated with a time interval that must have passed since the expulsion of the particle. The full quantum picture is more complicated due to the uncertainty of the momentum and the dispersion of the associated wave packet. The general picture, however, still holds, see the discussion in [45, Sect. 2.1].

The continuing decay of the wave function according to (41) means that the reference density of the bound part of the system decreases exponentially in time. Consequently the resonance wave function in its asymptotic part must grow exponentially in space, since more distant points in space are linked to earlier times and thus to larger initial densities.

This poses no problem in the case of a single channel, since the normalization with the integrated density accounts for exactly this effect. This is the physical reason why the ratio in (42) is well behaved. In the case of several open channels we face a challenge: Since the total energy, that is of course constant and dictated by the real part of the resonance, is distributed differently to system and expelled particle for the different channels, the escaping particles have different velocities and therefore the partial currents grow exponentially with different rates. In this sense points in the asymptotic region mix contributions from the different channels at different times. Since the density normalization is such that the total rate stays constant, it follows that the channel with the strongest exponential growth will dominate for $R \rightarrow \infty$, while all the others will vanish. This does not mean, that the partial rates in the physical sense depend on $R$, but rather, that a correction for the time mixing must be taken into account.

More precisely: If the channel potentials are asymptotically flat the rate of growth in space for channel $n$ is given asymptotically as

$$
-\operatorname{Im} \sqrt{2\left(E_{\text {res }}-E_{n}\right)}
$$

where $E_{\text {res }}$ is the (complex) eigenvalue of the resonance in question and $E_{n}$ is the (real) energy of the corresponding channel threshold. If this simple exponential behaviour can be shown in the computed data, then it provides a path to trace the flux of the ionized electron back to the bound region, from whence it must have been emitted. As anticipated above, however, a quantitatively correct procedure to trace back the fluxes would have to take into account to some extent at least the shape of the channel potentials before the asymptotics are reached and the initial probability distribution. The procedure suggested by equation (29) of Ref. [76], which implies tracing back the fluxes to the origin $\boldsymbol{r}=\mathbf{0}$ with linear velocities, might prove insufficient in this respect.

In this paper, however, we adopt an even simpler approach. We will see that in our case, even for rather large decay rates, the correction induced by different rates of exponential growth in space is in fact so small that we can neglect it (see Tables 1,2).

\section{Partial autoionisation rates in $1 \mathrm{D}$ helium}

In this section we apply the method developed in Section 3 to the one dimensional helium.

\subsection{General formulation}

After single ionization of the helium atom the remaining ion resembles a hydrogen atom with different nuclear charge $Z$. For this system the states are known (see for 
example $[113,114])$ and are given as

$$
\phi_{N}(r)=\frac{\sqrt{Z}}{N} \frac{1}{\sqrt{N}} \exp \left(-\frac{Z r}{N}\right)\left(\frac{2 Z r}{N}\right) L_{N-1}^{(1)}\left(\frac{2 Z r}{N}\right),
$$

where $Z$ is the nuclear charge, $N$ is the principal quantum number of the electron and $r$ its distance from the nucleus. Using Sturmian functions as defined in (17) this writes as

$$
\langle r \mid N\rangle:=\phi_{N}(r) \equiv(-1)^{N} \frac{\sqrt{Z}}{N} S_{N}^{\left(\frac{N}{Z}\right)}(r) .
$$

We can use the states of the remaining ion to define different decay channels. Since the outer electron has escaped the system, it is expected to behave like a Coulomb wave. Furthermore we do not care about its energy. Following Sec. 2.4 we restrict our analysis to the region $z_{1}>z_{2}$. Here, $z_{1}$ is the coordinate of the outer electron and in accordance with (28) we can define decay channels by the projection operators

$$
P_{N}=\mathbb{1} \otimes|N\rangle\langle N| .
$$

In the case of $e Z e$ we can execute the projection using the orthogonality of the Sturmian functions as

$$
\begin{aligned}
P_{N}|\psi\rangle & =(\mathbb{1} \otimes|N\rangle\langle N|) \sum_{n m} C_{n m}\left|S_{n}^{(\alpha)}\right\rangle \otimes\left|S_{m}^{(\beta)}\right\rangle \\
& =\sum_{n m} C_{n m}\left\langle N \mid S_{m}^{(\beta)}\right\rangle\left|S_{n}^{(\alpha)}\right\rangle \otimes|N\rangle,
\end{aligned}
$$

where $\left\langle N \mid S_{m}^{(\beta)}\right\rangle$ can be calculated as

$$
\left\langle N \mid S_{m}^{(\beta)}\right\rangle=(-1)^{N} \frac{\sqrt{Z}}{N}\left\langle\tilde{S}_{N}^{\left(\frac{N}{Z}\right)}\left|z_{2}\right| S_{m}^{(\beta)}\right\rangle,
$$

which in turn can be computed from (38) with a complex argument, thus reducing the projection to a simple matrix vector multiplication.

In the Zee case this approach poses a severe challenge: By introducing perimetric coordinates, we found an efficient basis that automatically guarantees proper boundary conditions. Unfortunately this also mixes the coordinates of the two electrons. Therefore, the product structure of our basis is not the product of two one-electron spaces, and the found eigenvectors cannot be projected easily. For these reasons, we resort to projection by integration on a grid in coordinate space, defining the aforementioned projector by

$$
P_{N} \psi_{>}\left(z_{1}, z_{2}\right):=\phi_{N}\left(z_{2}\right) \int_{0}^{\infty} \phi_{N}\left(z_{2}^{\prime}\right) \psi_{>}\left(z_{1}, z_{2}^{\prime}\right) \mathrm{d} z_{2}^{\prime} .
$$

Following the treatment laid out in Section 3 we choose the volume $V_{R}$ quadratic as

$$
V_{R}=\left\{\left(z_{1}, z_{2}\right) \in \mathbb{R}^{2} \mid 0 \leq z_{1} \leq R ; 0 \leq z_{2} \leq R\right\} .
$$

Since the wave function in our case lives completely inside this region for $R \rightarrow \infty$, clearly there can be no flux through the $z_{1}$ nor the $z_{2}$ axis. Consequently, for any fixed, finite $R$, the flux out of $V_{R}$ is given by the two contributions of current through the boundary $\left\{\left(R, z_{2}\right) \mid z_{2} \leq R\right\}$ and $\left\{\left(z_{1}, R\right) \mid z_{1} \leq R\right\}$. Due to the symmetry of the problem they must be identical. Furthermore the enclosed density must, for the same reason, be distributed equally among the two triangular parts of $V_{R}$ defined by the diagonal. Hence it is enough to look at the triangular region $\left\{\left(z_{1}, z_{2}\right) \in \mathbb{R}^{2} \mid 0 \leq z_{1} \leq\right.$ 
$\left.R ; 0 \leq z_{2} \leq z_{1}\right\}$. The flux out of $V_{R}$ is given only by the flux through the $z_{1}=R$ boundary. On this line, the perpendicular direction is the $z_{1}$ direction, and therefore the relevant current density operator is given as

$$
\tilde{J}\left(z_{1}, z_{2}\right)=\frac{1}{2}\left[p_{z_{1}}\left|z_{1}, z_{2}\right\rangle\left\langle z_{1}, z_{2}|+| z_{1}, z_{2}\right\rangle\left\langle z_{1}, z_{2}\right| p_{z_{1}}\right] .
$$

Since $\tilde{J}$ only acts in direction of $z_{1}$, and $P_{N}$ only in direction of $z_{2}$, we have $\left[P_{N}, \tilde{J}\right]=0$. This guarantees the vanishing of the second term in (50), and in this sense the choice of $V_{R}$ is compatible with the given channels.

Therefore, the relevant component of the partial flux $j_{N}\left(z_{1}, z_{2}\right)$ can be calculated exactly like the total flux $j\left(z_{1}, z_{2}\right)$, with the projected wave function $\psi_{N}$ instead of the total wave function $\psi$ :

$$
\tilde{j}_{(N)}\left(z_{1}, z_{2}\right)=\operatorname{Im}\left[\psi_{(N)}^{*}\left(z_{1}, z_{2}\right) \frac{\partial}{\partial z_{1}} \psi_{(N)}\left(z_{1}, z_{2}\right)\right]
$$

Thanks to the properties of the Laguerre polynomials, both factors can be calculated without numeric approximation of the derivative. Furthermore, since $P_{N}$ only acts on $z_{2}$ and the derivative is in direction of $z_{1}$, the two operators commute and we can calculate $\frac{\partial}{\partial z_{1}} \psi_{N}\left(z_{1}, z_{2}\right)$ by projection of $\frac{\partial}{\partial z_{1}} \psi\left(z_{1}, z_{2}\right)$. In this setup the role of $R$ is assumed by $z_{1}$. Where no confusion is possible we will write $z_{1}$ also for $R$ in the following.

\subsection{Selected resonances}

In this section we demonstrate some results on relatively low lying, doubly excited states, with both electrons similarly excited. We use the $(4,6)$ resonance as a prototypical example for the Zee configuration. For its density and numerical parameters see Figure 4. As stated earlier, the full resonance wave function is not normalizable. Our numeric approximation, however, due to the finite extension of our basis, is normalizable. Since a global factor does not change the equations, we normalize it to $\lim _{z_{1} \rightarrow \infty} D\left(z_{1}\right)=1$.

The current density that has to be integrated is shown in Figures 6 and 7. The strong circular currents in the bound region necessitate a particularly careful integration. This is carried out with the adaptive Gauss-Kronrod method as provided by the GNU Scientific Library. Note that the current density in the asymptotic region is much better behaved and consequently easier to integrate. 


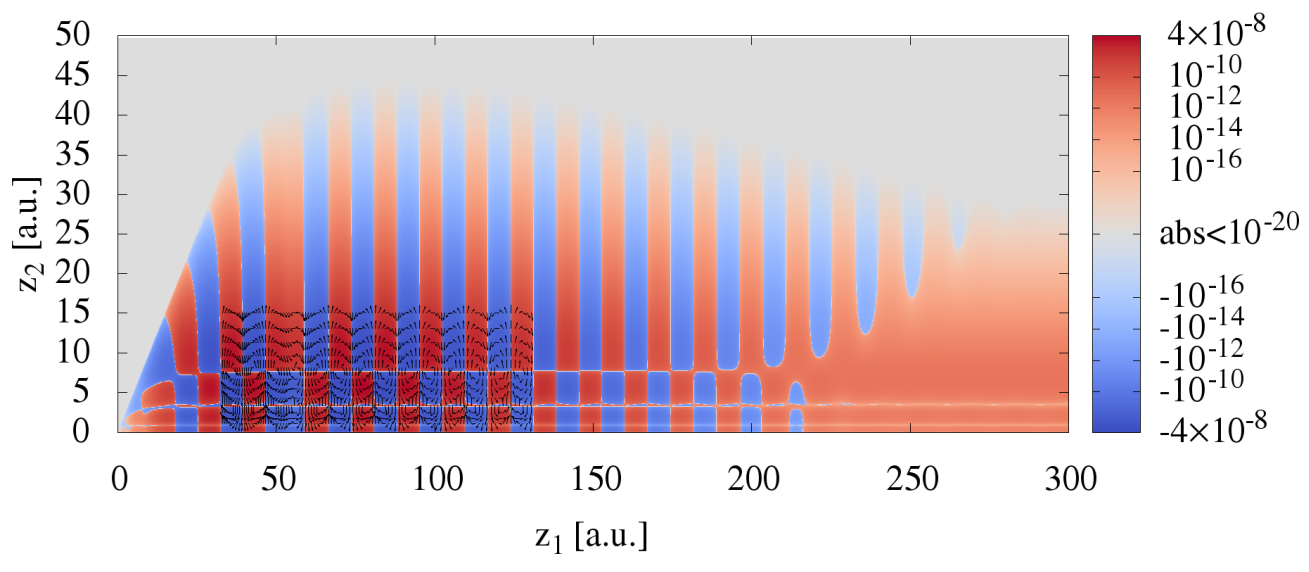

Figure 6. Current density in direction of $z_{1}$ for the Zee $(4,6)$ resonance. Grey marks all areas with a magnitude smaller than $10^{-20}$ a.u. Positive values (that is flux in positive $z_{1}$ direction, i.e. outgoing flux) are plotted with a red logarithmic color scale, negative values with a blue logarithmic color scale. The area shown is the same as for the probability density in Figure 4, that is the bound part and the onset of the asymptotic region. In the bound region a checkerboard pattern is observed, which can be attributed to circular currents. To illustrate this, part of the associated vector field is indicated in the range $z_{1} \in[30$ a.u., 125 a.u.]. The slight irregularity around $z_{1}=50$ a.u. coincides with the vertical nodal line of the probability density at the same position (c.f. Figure 4). Values are as large as $4 \cdot 10^{-8}$ a.u. in magnitude, with several contributions to the integral in (53) canceling each other to arrive at the much smaller rate $\Gamma=1.41328 \cdot 10^{-11}$ a.u. Observe that, for $z_{1} \gtrsim 150$ a.u., the current, while still oscillating, has already decreased to a magnitude which is of the same order as in the asymptotic region. Compare this with the noise in Figure 8. The transition region, starting around $z_{1} \gtrsim 200$ a.u., and the subsequent onset of the asymptotic region are shown in more detail in Figure 7.

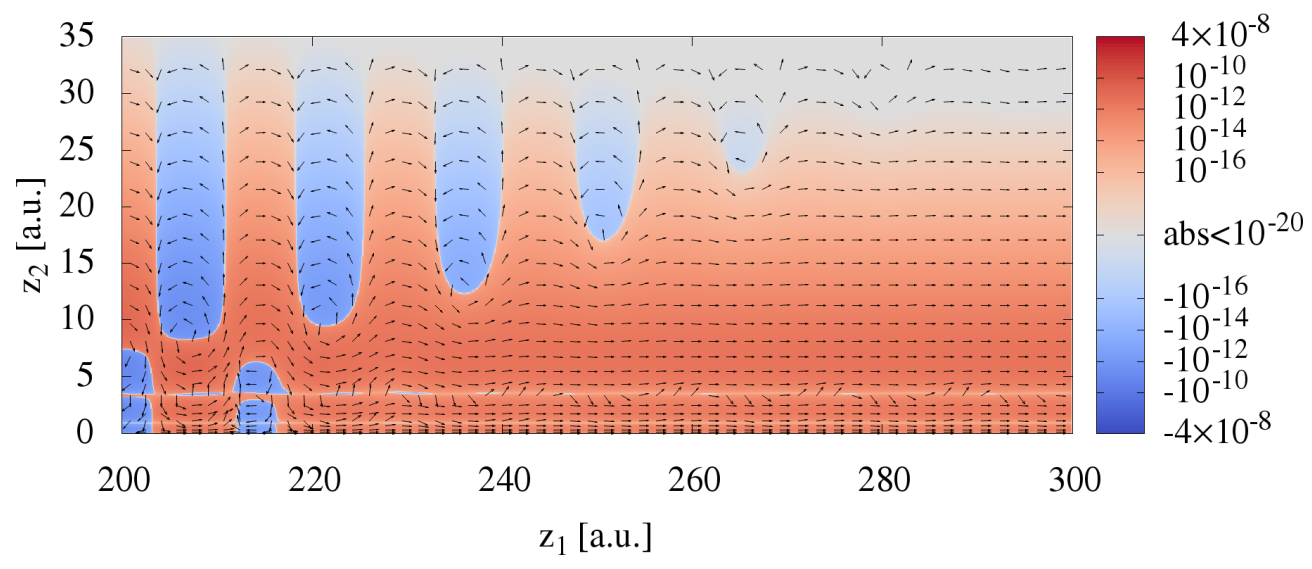

Figure 7. Current density in direction of $z_{1}$ for the Zee $(4,6)$ resonance (color code) and direction of current (arrows). Shown is a closeup of the transition region from the bound to the asymptotic region. On the left, around $z_{1} \approx 200$ a.u., the current behaviour as observed in the checkerboard region of Figure 6 can be seen. Strong circular currents fill that area. As $z_{1}$ increases the circular currents disappear and a mostly parallel outward current prevails. 
Figure 8 shows the resulting integrated quantities $\gamma$ and $D,(52,53)$, for the $(4,6)$ resonance. $D$ quickly reaches 1 . This is because the bulk of the density is in the bound region, where the asymptotic regions only contribute very small amounts (cf Fig. 4). We see that $\gamma$ follows the shape of $D$. Figure 9 shows the normalized current with the rate as calculated from the eigenvalue superimposed. Even deep into the bound part the calculations agree. Only very close to the origin numerical problems dominate.

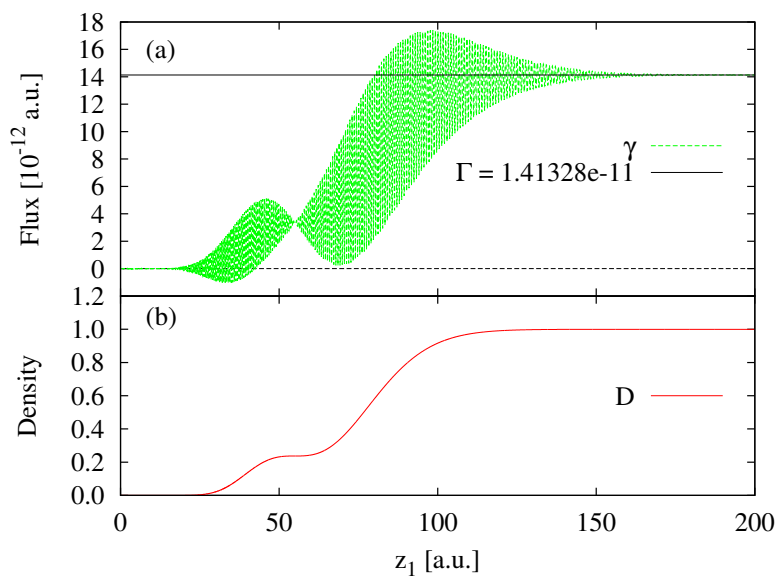

Figure 8. Total current in direction of $z_{1}$ (a) and integrated density (b) for the Zee $(4,6)$ resonance (c.f. Figure 4).

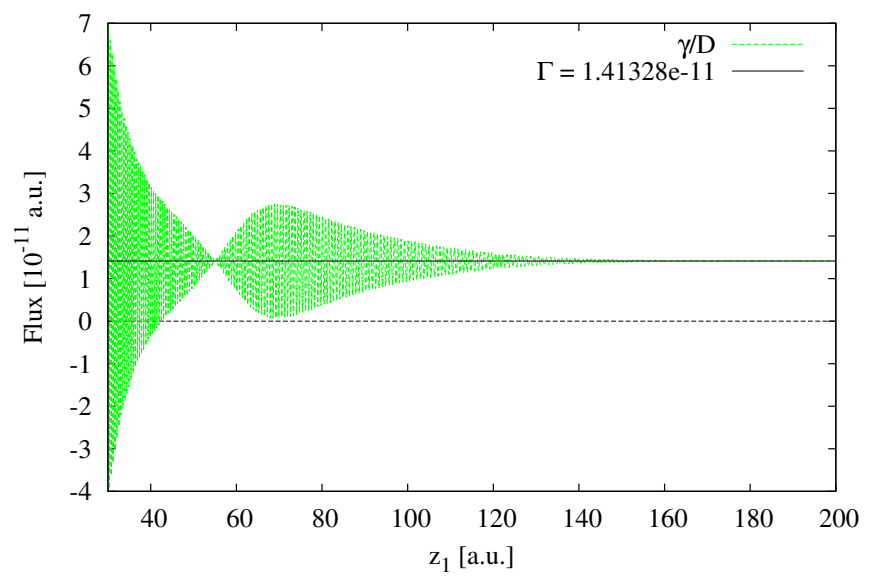

Figure 9. Total current, normalized by the integrated density for the Zee $(4,6)$ resonance. On average it agrees with the rate as given by the eigenvalue calculation even in the bound part. Very close to the origin both numerator and denominator become small, leading to numerical problems. 
Let us take a look at the total current in the asymptotic region. This is shown in Figure 10. While far away from the nucleus also numerical problems increase, the current calculation agrees with the rate from the eigenvalue calculation for a wide range of configuration space.

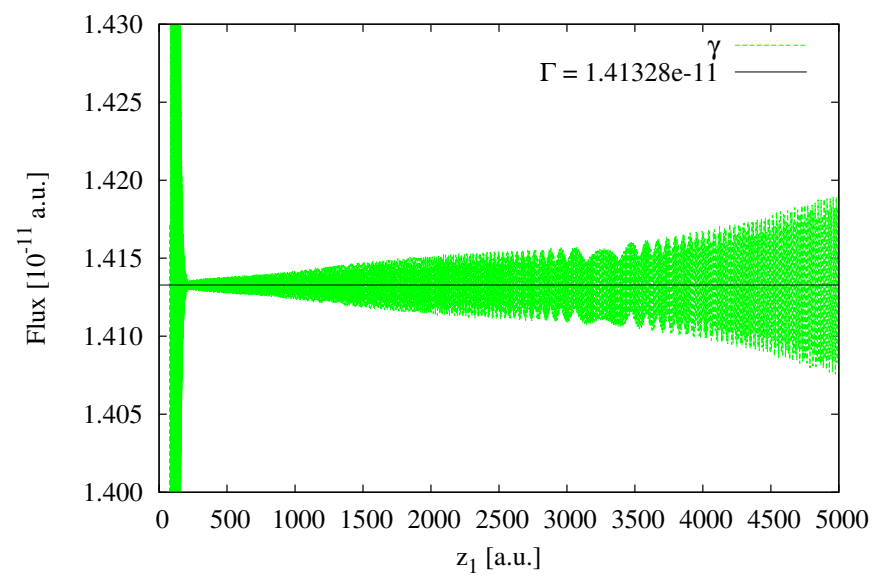

Figure 10. Total current in the asymptotic region for the Zee $(4,6)$ resonance. The average value agrees with the rate known from the eigenvalue calculation over a wide region of configuration space. Even at large distances from the nucleus the noise level is below 1 per cent. 
We now turn to the analysis of partial rates. Figure 11 shows the partial currents in all energetically possible channels in the bound region. We see how the two dominant channels interact. Their amplitude is three orders of magnitude larger than the total current, to which they add up. Here we do not show the normalized currents $\gamma / D$, but their unnormalized counterparts. This is done to avoid numerical problems and does not alter the analysis, in that it would, at every point, only add a factor that is constant for all channels.

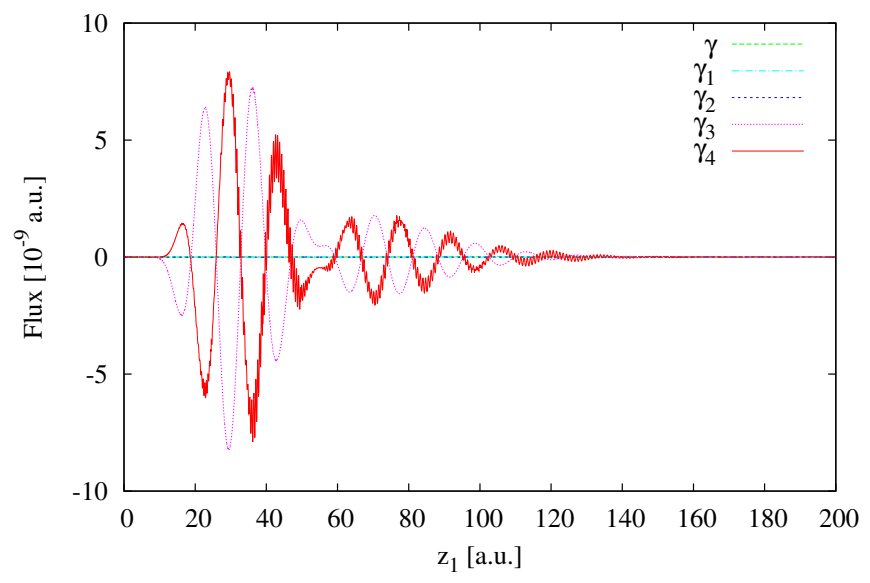

Figure 11. Partial currents of the Zee $(4,6)$ resonance for all open channels in the bound region of configuration space. The two most dominant contributions are seen to cancel each other despite significant oscillations. The interplay of the partial rates indicates that the channels are not yet separated.

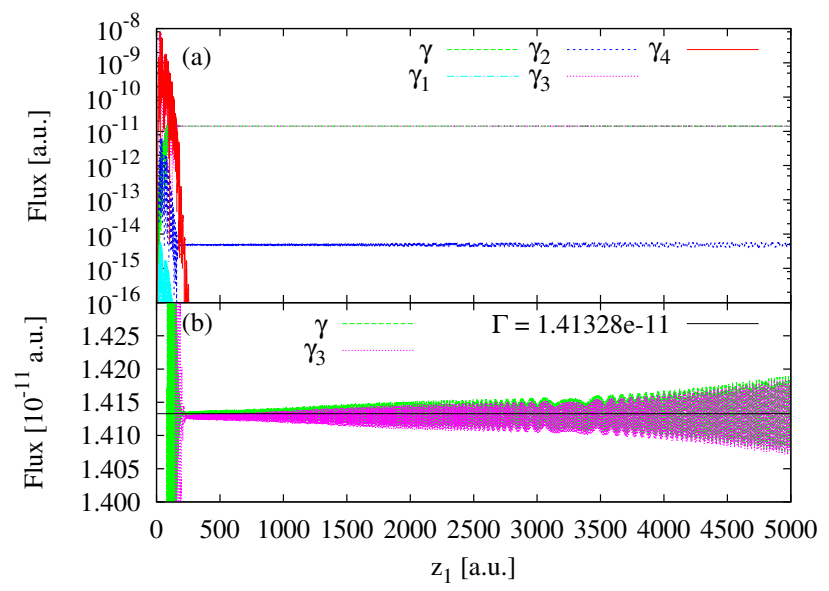

Figure 12. Partial currents of the Zee $(4,6)$ resonance for (a) all open channels in the asymptotic regime, (b) the dominant channel. In the logarithmic plot (a) we see $\gamma_{1}$ and $\gamma_{4}$ quickly drop to values which, due to the finite precision arithmetics, need to be considered as numerically zero. The dominant contribution to the total current stems from the $\gamma_{3}$ component, with a small contribution from $\gamma_{2}$. The linear plot (b) confirms this. 
Table 1. Comparison of the rates for the $(4,6)$ state of Zee helium as obtained from eigenvalue $(\Gamma)$ and current calculations. The latter are calculated as the averages over part of the asymptotic region which, from the current plots (see Figure 12), is determined as the interval $z_{1} \in[200$ a.u., 5000 a.u. $]$.

\begin{tabular}{ccc} 
Source & Rate & Standard deviation \\
\hline$\Gamma$ & $1.41328 \cdot 10^{-11}$ a.u. & \\
$\gamma$ & $1.41328 \cdot 10^{-11}$ a.u. & $1.4 \cdot 10^{-14}$ a.u. \\
$\gamma_{3}$ & $1.41279 \cdot 10^{-11}$ a.u. & $1.4 \cdot 10^{-14}$ a.u. \\
$\gamma_{2}$ & $0.00049 \cdot 10^{-11}$ a.u. & $3.0 \cdot 10^{-16}$ a.u. \\
$\gamma_{3}+\gamma_{2}$ & $1.41328 \cdot 10^{-11}$ a.u. & $1.5 \cdot 10^{-14}$ a.u.
\end{tabular}

Figure 12 shows the asymptotic behaviour of the partial currents. Channels $N=1,4$ quickly drop to numerically zero. Channels $N=2,3$ become constant and clearly separate. Indeed this justifies the notion of an asymptotic region directly from the data without an ad hoc definition.

The $N=3$ channel is the dominant one. The only other contribution is from the $N=2$ channel. We estimate partial rates as the average values of the partial currents in the interval $z_{1} \in[200$ a.u., 5000 a.u.]. The results are shown in Table 1. Note, in particular, the high accuracy of our approach, which is reflected in the agreement of the sum of partial rates with the total rate in 6 significant digits. Clearly in this case the correction for the exponential growth of the partial currents that was discussed in Section 3.2 is not necessary. This could be due to the rather small rates. Next we examine a case with much stronger decay.

We now present results for the $e Z e$ configuration using the $(4,7$, even) resonance as an example. Its probability density has already been shown in Figure 5. Figure 13 shows the total current prior to normalization together with the integrated density. Both exhibit the expected structured increase in the bound region with $z_{1}<100$ a.u. Contrary to the Zee case (cf. Figure 8) the asymptotic region is not flat, but is in fact increasing exponentially. We therefore make no attempt to normalize the state

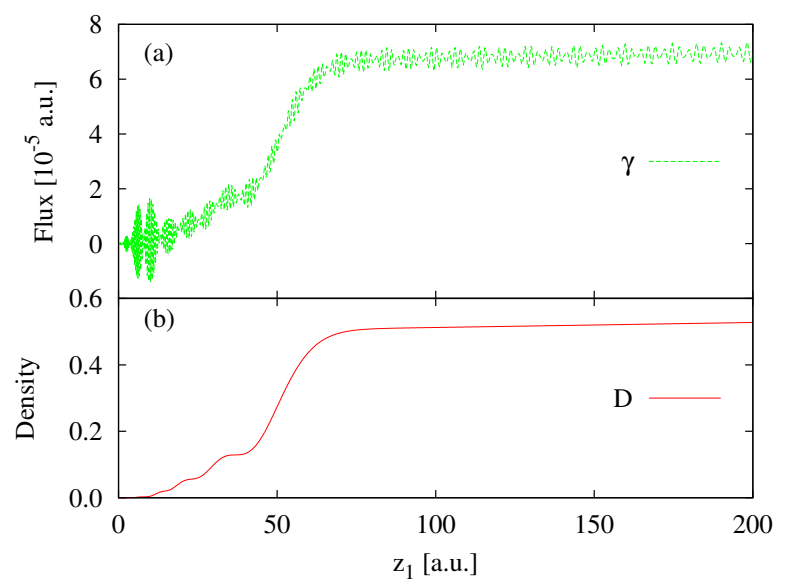

Figure 13. Total current in direction of $z_{1}$ (a) and integrated density (b) for the $e Z e(4,7$, e) resonance (c.f. Figure 5). 


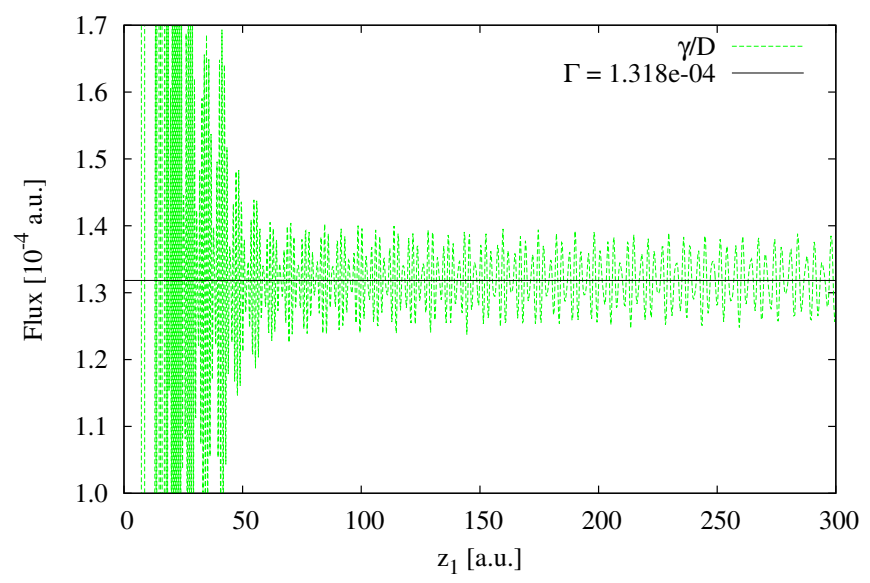

Figure 14. Total current, normalized by the integrated density for the $e Z e$ $(4,7, \mathrm{e})$ resonance. On average it agrees with the rate as given by the eigenvalue calculation even in the bound part. Close to the origin both numerator and denominator become small, leading to numerical problems.

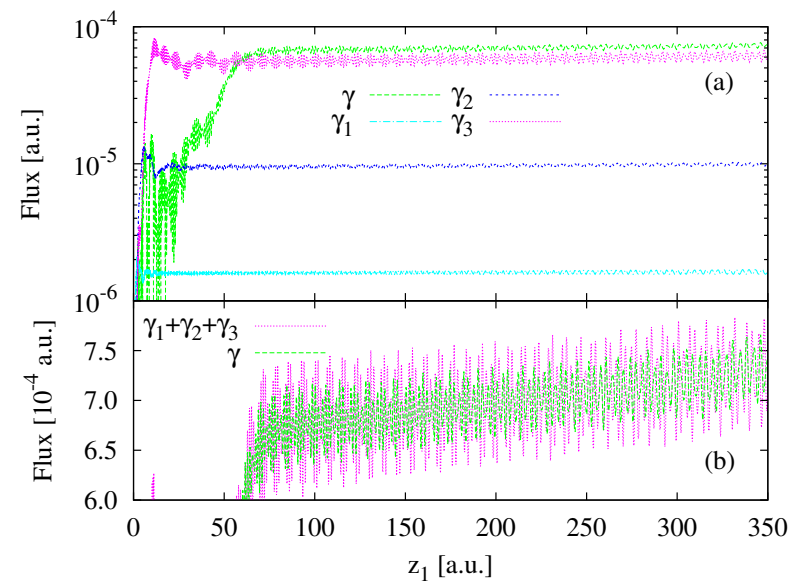

Figure 15. Partial currents of the $e Z e(4,7, \mathrm{e})$ resonance for (a) all contributing channels in the asymptotic regime, (b) the sum of all contributing channels compared to the total current. In the logarithmic plot (a) we see that three channels, $\gamma_{1}, \gamma_{2}$, and $\gamma_{3}$, contribute with increasing relevance. The linear plot (b) shows the agreement of the sum of partial currents and total current and the expected growth with increasing $z_{1}$. This is corrected for by the integrated density (c.f. Figure 16).

such that the integrated density is 1 . Consequently all not normalized currents are practically in arbitrary units. Figure 14 demonstrates, that the normalized total current again agrees with the decay rate as calculated from the eigenvalue. The exponential increases of current and density cancel and give a proper constant rate, save for some variation introduced by numerics. We now turn to the analysis of partial rates. The partial currents without normalization are shown in Figure 15. In part (a) 


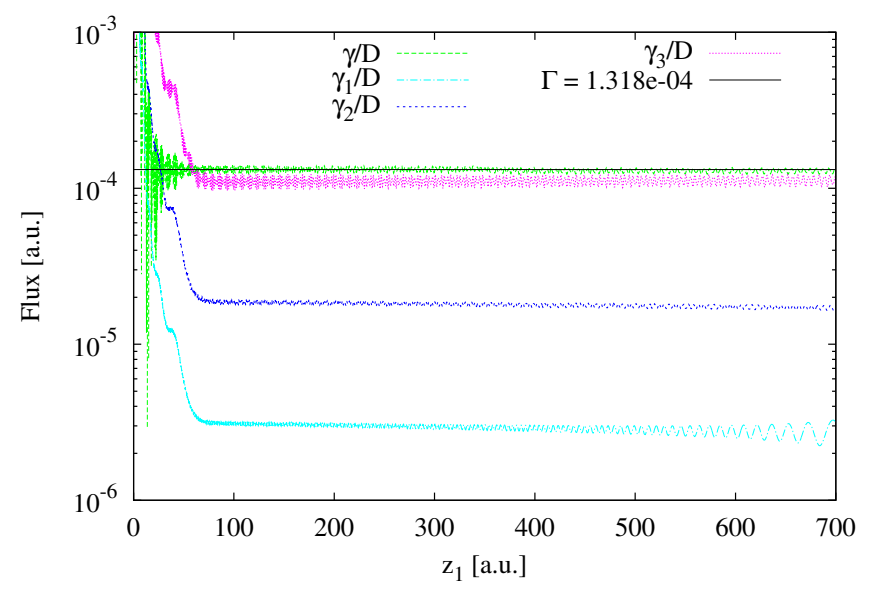

Figure 16. Partial currents of the $e Z e(4,7, \mathrm{e})$ resonance, normalized by the integrated density. See Figure 15 for the agreement of partial and total currents. The total rate as calculated from the eigenvalue is overlaid. While the curves are not constant, even on average in the asymptotic regime, they change so little as to permit the calculation of a meaningful average (cf discussion at the end of Section 3.2). The results are shown in Table 2.

we see that all three open channels have partial currents of non negligible magnitude. Part (b) shows that their sum indeed reflects the total current. This allows us to understand the ripples that have been shown in the probability density of the state (Figure 5): The shape of the outgoing part in ionic channel $N$ is characterized by $N$ antinodes in $z_{2}$ direction. Hence the antinode closest to the $z_{1}$ axis is occupied by all three outgoing channels, the second one by the $N=2,3$ channels and the third one only by the $N=3$ channel. In $z_{1}$ direction the wave functions resemble Coulomb waves with different momenta $k$ as determined by the differences in energy between the corresponding ionic state of the remaining system and the initial energy of the resonance. These different momenta produce a beating effect that shows up as ripples in the density.

For a quantitative analysis of the partial currents we turn to Figure 16. It contains the normalized partial and total currents together with the total rate as calculated from the eigenvalue. From this we fit constants for the partial rates in the asymptotic region, here taken as $z_{1} \in[100$ a.u., 700 a.u.]. The results are shown in Table 2. Even with large decay rates and several contributing channels as in this case, the partial rates agree with the total rate. Moreover, despite the noticeable exponential growth, in a large enough region of configuration space that still certainly can be called asymptotic, they are approximately constant.

\section{Conclusion}

We have presented a theoretical framework for the study of partial decay rates. Partial decay channels can be resolved on the basis of projection operators of wave functions. This was combined with the method of complex rotation to allow for high precision analysis of resonances. For the first time, partial rates have been calculated without 
Table 2. Comparison of the rates as obtained from eigenvalue $(\Gamma)$ and current calculations. The latter are calculated as the averages over part of the asymptotic region which, from the current plots (see Figure 12), is determined as the interval $z_{1} \in[100$ a.u., 700 a.u. $]$.

\begin{tabular}{ccc} 
Source & Rate & Standard deviation \\
\hline$\Gamma$ & $1.3183 \cdot 10^{-4}$ a.u. & \\
$\gamma$ & $1.3202 \cdot 10^{-4}$ a.u. & $1.4 \cdot 10^{-7}$ a.u. \\
$\gamma_{3}$ & $1.1109 \cdot 10^{-4}$ a.u. & $1.5 \cdot 10^{-7}$ a.u. \\
$\gamma_{2}$ & $0.17970 \cdot 10^{-4}$ a.u. & $1.3 \cdot 10^{-8}$ a.u. \\
$\gamma_{1}$ & $0.02928 \cdot 10^{-4}$ a.u. & $3 \cdot 10^{-9}$ a.u. \\
$\gamma_{3}+\gamma_{2}+\gamma_{1}$ & $1.3198 \cdot 10^{-4}$ a.u. & $1.7 \cdot 10^{-7}$ a.u.
\end{tabular}

the assumption of a specific final state from complex rotation. The utility of the method has been demonstrated by its application to two one-dimensional helium model systems. Our approach was successful, despite the radically different rates, for both the extremely long-lived Zee configuration and the $e Z e$ case, which exhibits strong decay. Notwithstanding the long-range nature of the involved Coulomb potential, an asymptotic regime was clearly observed for both situations. The approach can be applied to other decay problems like higher dimensional helium. Here, building on earlier work in which backrotated wave functions have already been calculated [115], what remains to be done is the definition of proper channel projectors. This endeavour seems to be promising in the light of the total rates that have been compared for 1D, 2D, and 3D helium [52, Figs. 2,3] and that were found to be on the same order for 2D, $3 \mathrm{D}$ and $1 \mathrm{D}$ eZe rates, while the $1 \mathrm{D}$ Zee rates are much smaller as also demonstrated here.

\section{Acknowledgements}

We acknowledge the use of the computing resources provided by the Black Forest Grid Initiative, by bwGRiD (http://www.bw-grid.de), and the computing time granted by the John von Neumann Institute for Computing (NIC), and alotted on the supercomputer JUROPA at Jülich Supercomputing Centre (JSC). Furthermore, funding by DFG Research Unit 760 is gratefully acknowledged. P. L. acknowledges financial support from the Humboldt Foundation through Fellowship No. 1139948.

\section{References}

[1] Tanner G, Richter K and Rost J M 2000 Rev. Mod. Phys. 72 497-544

[2] Richter K and Wintgen D 1990 J. Phys. B: At. Mol. Opt. Phys. 23 L197

[3] Jiang Y H, Püttner R, Delande D, Martins M and Kaindl G 2011 Phys. Rev. A 84023402

[4] Fano U 1961 Phys. Rev. 124 1866-1878

[5] Madden R P and Codling K 1963 Phys. Rev. Lett. 10 516-518

[6] Pisharody S N and Jones R R 2004 Science 303 813-815

[7] Eiglsperger J, Piraux B and Madroñero J 2010 Phys. Rev. A 81042527

[8] Walker B, Sheehy B, DiMauro L F, Agostini P, Schafer K J and Kulander K C 1994 Phys. Rev. Lett. 73 1227-1230 
[9] Byun C W, Choi N N, Lee M H and Tanner G 2007 Phys. Rev. Lett. 98113001

[10] Becker W and Rottke H 2008 Contemp. Phys. 49 199-223

[11] Palacios A, Horner D A, Rescigno T N and McCurdy C W 2010 J. Phys. B: At. Mol. Opt. Phys. 43194003

[12] Jörder F, Zimmermann K, Rodriguez A and Buchleitner A 2014 Phys. Rev. Lett., accepted for publication (arXiv:1311.5742)

[13] Fano U 1983 Rep. Prog. Phys. 4697

[14] Leopold J G and Percival I C 1980 J. Phys. B: At. Mol. Phys. 131037

[15] Feagin J M and Briggs J S 1986 Phys. Rev. Lett. 57 984-987

[16] Briggs J S 1992 Nuclear Physics A 543 127-141

[17] Gutzwiller M C 1990 Chaos in Classical and Quantum Mechanics (Springer) ISBN 9780387971735

[18] Ezra G S, Richter K, Tanner G and Wintgen D 1991 J. Phys. B: At. Mol. Opt. Phys. 24 L413

[19] Choi N N, Lee M H and Tanner G 2004 Phys. Rev. Lett. 93054302

[20] Sano M M and Tanikawa K 2008 Physics Letters A 372 6899-6903

[21] Keshavamurthy S and Schlagheck P 2011 Dynamical Tunneling: Theory and Experiment (Taylor \& Francis) ISBN 9781439816653

[22] Bürgers A, Wintgen D and Rost J M 1995 J. Phys. B: At. Mol. Opt. Phys. 28 3163

[23] Herrick D R, Kellman M E and Poliak R D 1980 Phys. Rev. A 22 1517-1535

[24] Herrick D R 1983 New symmetry properties of atoms and molecules Advances in Chemical Physics vol 52 ed Prigogine I and Rice S A (John Wiley \& Sons, Inc.) p 1-115 ISBN 9780470142769

[25] Macek J 1968 J. Phys. B: At. Mol. Phys. 1831

[26] Lin C D 1984 Phys. Rev. A 29 1019-1033

[27] Lin C D 1995 Physics Reports 257 1-83

[28] Feagin J M and Briggs J S 1988 Phys. Rev. A 37 4599-4613

[29] Rost J M and Briggs J S 1991 J. Phys. B: At. Mol. Opt. Phys. 244293

[30] Domke M, Schulz K, Remmers G, Kaindl G and Wintgen D 1996 Phys. Rev. A 53 1424-1438

[31] Fittinghoff D N, Bolton P R, Chang B and Kulander K C 1992 Phys. Rev. Lett. $692642-2645$

[32] Ott C, Kaldun A, Raith P, Meyer K, Laux M, Zhang Y, Hagstotz S, Ding T, Heck R and Pfeifer T 2012 Quantum interferometry and correlated two-electron wave-packet observation in helium arXiv e-print 1205.0519

[33] Rost J M and Briggs J S 1990 J. Phys. B: At. Mol. Opt. Phys. 23 L339

[34] Rost J M, Schulz K, Domke M and Kaindl G 1997 J. Phys. B: At. Mol. Opt. Phys. 304663

[35] Rost J M, Gersbacher R, Richter K, Briggs J S and Wintgen D 1991 J. Phys. B: At. Mol. Opt. Phys. 242455

[36] Lambropoulos P, Maragakis P and Zhang J 1998 Physics Reports 305 203-293 
[37] Aguilar J and Combes J M 1971 Comm. Math. Phys. 22 269-279

[38] Balslev E and Combes J M 1971 Comm. Math. Phys. 22 280-294

[39] Simon B 1972 Comm. Math. Phys. 27 1-9

[40] Simon B 1973 Annals of Mathematics 97 247-274

[41] Simon B 1978 Int. J. Quantum Chem. 14 529-542

[42] Reed M and Simon B 1978 Methods of modern mathematical physics: Analysis of operators (Academic Press) ISBN 9780125850049

[43] Reinhardt W P 1982 Annu. Rev. Phys. Chem. 33 223-255

[44] Junker B 1982 Recent computational developments in the use of complex scaling in resonance phenomena Advances in Atomic and Molecular Physics vol Volume 18 ed Bates D and Bederson B (Academic Press) pp 207-263 ISBN 0065-2199

[45] Ho Y 1983 Physics Reports 99 1-68

[46] Lindroth E 1994 Phys. Rev. A 49 4473-4480

[47] Moiseyev N 1998 Physics Reports 302 212-293

[48] Buchleitner A, Delande D and Gay J C 1995 J. Opt. Soc. Am. B 12 505-519

[49] Buchleitner A, Grémaud B and Delande D 1994 J. Phys. B: At. Mol. Opt. Phys. 272663

[50] Wintgen D and Delande D 1993 J. Phys. B: At. Mol. Opt. Phys. 26 L399

[51] Grémaud B and Delande D 1997 EPL 40363

[52] Madroñero J, Schlagheck P, Hilico L, Grémaud B, Delande D and Buchleitner A 2005 EPL $\mathbf{7 0} 183$

[53] Eiglsperger J and Madroñero J 2009 Phys. Rev. A 80022512

[54] Scrinzi A and Piraux B 1997 Phys. Rev. A 56 R13-R16

[55] Foumouo E, Kamta G L, Edah G and Piraux B 2006 Phys. Rev. A 74063409

[56] Nikolopoulos L a A and Lambropoulos P 2007 J. Phys. B: At. Mol. Opt. Phys. 401347

[57] Hochstuhl D and Bonitz M 2011 J. Chem. Phys. 134084106

[58] Burke P G and Taylor K T 1975 J. Phys. B: At. Mol. Phys. 82620

[59] Hayes M A and Scott M P 1988 J. Phys. B: At. Mol. Opt. Phys. 211499

[60] Malegat L, Selles P and Kazansky A K 1999 Phys. Rev. A 60 3667-3676

[61] Selles P, Malegat L and Kazansky A K 2002 Phys. Rev. A 65032711

[62] Sánchez I and Martín F 1993 Phys. Rev. A 48 1243-1251

[63] Tang J Z, Watanabe S and Matsuzawa M 1992 Phys. Rev. A 46 2437-2444

[64] Tang J Z, Watanabe S, Matsuzawa M and Lin C D 1992 Phys. Rev. Lett. 69 $1633-1635$

[65] Tang J Z and Shimamura I 1994 Phys. Rev. A 50 1321-1327

[66] Bray I, Fursa D V, Kheifets A S and Stelbovics A T 2002 J. Phys. B: At. Mol. Opt. Phys. 35 R117

[67] Menzel A, Frigo S P, Whitfield S B, Caldwell C D, Krause M O, Tang J Z and Shimamura I 1995 Phys. Rev. Lett. 75 1479-1482

[68] Menzel A, Frigo S P, Whitfield S B, Caldwell C D and Krause M O 1996 Phys. Rev. A 54 2080-2090 
[69] Bouri C, Selles P, Malegat L and Kwato Njock M 2007 J. Electron. Spectrosc. Relat. Phenom. 161 80-84

[70] Rescigno T N, Baertschy M, Isaacs W A and McCurdy C W 1999 Science 286 2474-2479

[71] Noro T and Taylor H S 1980 J. Phys. B: At. Mol. Phys. 13 L377

[72] Bačić Z and Simons J 1982 Int. J. Quantum Chem. 21 727-739

[73] Peskin U, Moiseyev N and Lefebvre R 1990 J. Chem. Phys. 922902

[74] Moiseyev N and Peskin U 1990 Phys. Rev. A 42 255-260

[75] Schmidt M 2009 Partial rates with complex dilation Diplomarbeit Albert-Ludwigs-University Freiburg URL http://http://www.freidok. uni-freiburg.de/volltexte/8075/

[76] Goldzak T, Gilary I and Moiseyev N 2010 Phys. Rev. A 82052105

[77] Ostrovsky V N and Prudov N V 1995 J. Phys. B: At. Mol. Opt. Phys. 284435

[78] Schlagheck P and Buchleitner A 1998 J. Phys. B: At. Mol. Opt. Phys. 31 L489

[79] Richter K and Wintgen D 1990 Phys. Rev. Lett. 65 1965-1965

[80] Baylis W and Drake G 2006 Units and constants Springer Handbook of Atomic, Molecular, and Optical Physics ed Drake G (Springer New York) pp 1-6 ISBN 978-0-387-20802-2, 978-0-387-26308-3

[81] Richter K, Tanner G and Wintgen D 1993 Phys. Rev. A 48 4182-4196

[82] Richter K and Wintgen D 1991 J. Phys. B: At. Mol. Opt. Phys. 24 L565

[83] Schlagheck P 1999 Das Drei-Körper-Coulombproblem unter periodischem Antrieb Ph.D. thesis Technische Universität München URL http://orbi.ulg. ac. be/handle/2268/75146

[84] Brotton S J, Cvejanovic S, Currell F J, Bowring N J and Read F H 1997 Phys. Rev. A 55 318-328

[85] Schlagheck P and Buchleitner A 2003 Eur. Phys. J. D 22 401-415

[86] Hochstuhl D, Balzer K, Bauch S and Bonitz M 2010 Physica E 42 513-519

[87] Doolen G D, Nuttal J and Stagat R W 1974 Phys. Rev. A 10 1612-1615

[88] Morse P M and Feshbach H 1953 Methods of theoretical physics (McGraw-Hill)

[89] Reed M and Simon B 1972 Methods of modern mathematical physics: Functional analysis (Academic Press) ISBN 9780125850018

[90] Moiseyev N 2011 Non-Hermitian Quantum Mechanics (Cambridge University Press) ISBN 9781139496995

[91] Maquet A, Chu S I and Reinhardt W P 1983 Phys. Rev. A 27 2946-2970

[92] Johnson B R and Reinhardt W P 1983 Phys. Rev. A 28 1930-1944

[93] Cohen-Tannoudji C, Dupont-Roc J and Grynberg G 2008 Atom - Photon Interactions: Basic Processes and Applications (Wiley) ISBN 9780471293361

[94] Dalibard J and Cohen-Tannoudji C 1985 J. Phys. B: At. Mol. Phys. 181661

[95] Rescigno T N and McKoy V 1975 Phys. Rev. A 12 522-525

[96] Coolidge A S and James H M 1937 Phys. Rev. 51 855-859

[97] Pekeris C L 1958 Phys. Rev. 112 1649-1658 
[98] Conference on Mathematical Tables (1954 ; Cambridge M 1972 Handbook of mathematical functions with formulas, graphs, and mathematical tables 9th ed (New York: Dover Publications) ISBN 0-486-61272-4

[99] Weniger E J 1985 J. Math. Phys. 26276

[100] Krug A 2001 Alkali Rydberg States in Electromagnetic Fields Ph.D. thesis Ludwig-Maximilians-Universität München URL http://edoc.ub. uni-muenchen.de/336/

[101] Delande D and Gay J C 1984 J. Phys. B: At. Mol. Phys. 17 L335

[102] Delande D 1988 Atomes de Rydberg en champs statiques intenses Ph.D. thesis Université Pierre et Marie Curie - Paris VI URL http://tel. archives-ouvertes.fr/tel-00011864

[103] Hernández V, Román J E and Vidal V 2005 ACM Trans. Math. Software 31 351-362

[104] Balay S, Brown J, Buschelman K, Gropp W D, Kaushik D, Knepley M G, McInnes L C, Smith B F and Zhang H 2013 PETSc Web page http://www.mcs.anl.gov/petsc

[105] Balay S, Gropp W D, McInnes L C and Smith B F 1997 Efficient management of parallelism in object oriented numerical software libraries Modern Software Tools in Scientific Computing ed Arge E, Bruaset A M and Langtangen H P (Birkhäuser Press) p 163-202

[106] Amestoy P R, Duff I S, L'Excellent J Y and Koster J 2001 SIAM J. Matrix Anal. 83 Appl. 23 15-41

[107] Amestoy P R, Guermouche A, L'Excellent J Y and Pralet S 2006 Parallel Computing 32 136-156

[108] Hernández V, Román J E, Tomás A and Vidal V 2007 Krylov-schur methods in SLEPc Tech. Rep. STR-7 Universitat Politècnica de València available at http://www.grycap.upv.es/slepc

[109] Englefield M J 1972 Group theory and the Coulomb problem (Wiley-Interscience)

[110] Cohen-Tannoudji C, Diu B and Laloë F 1997 Quantenmechanik vol 1 (Berlin: Walter de Gruyter) ISBN 3-11-013592-2

[111] Cohen-Tannoudji C, Diu B and Laloë F 2005 Quantum Mechanics vol 1 (Singapore: Wiley) ISBN 978-0-471-16433-3

[112] Argenti L, Pazourek R, Feist J, Nagele S, Liertzer M, Persson E, Burgdörfer J and Lindroth E 2013 Phys. Rev. A 87053405

[113] Landau L and Lifschitz E 1981 Course of Theoretical Physics Vol. 3: Quantum Mechanics (Oxford: Butterworth-Heinemann) ISBN 978-0750635394

[114] Blümel R and Smilansky U 1987 Z. Phys. D: Atom., Mol. Cl. 6 83-105

[115] Madroñero J and Buchleitner A 2008 Phys. Rev. A 77053402 